\title{
Meta-analysis of probability estimates of worldwide variation of CYP2D6 and CYP2C19
}

\author{
Anne B. Koopmans (10), Mario H. Braakman ${ }^{3,4}$, David J. Vinkers², Hans W. Hoek (1) ${ }^{1,5,6}$ and Peter N. van Harten ${ }^{2,7}$
}

\begin{abstract}
Extensive migration has led to the necessity of knowledge regarding the treatment of migrants with different ethnical backgrounds. This is especially relevant for pharmacological treatment, because of the significant variation between migrant groups in their capacity to metabolize drugs. For psychiatric medications, CYP2D6 and CYP2C19 enzymes are clinically relevant. The aim of this meta-analysis was to analyze studies reporting clinically useful information regarding CYP2D6 and CYP2C19 genotype frequencies, across populations and ethnic groups worldwide. To that end, we conducted a comprehensive meta-analysis using Embase, PubMed, Web of Science, and PsycINFO (>336,000 subjects, 318 reports). A non-normal metabolizer (non-NM) probability estimate was introduced as the equivalent of the sumprevalence of predicted poor, intermediate, and ultrarapid metabolizer CYP2D6 and CYP2C19 phenotypes. The probability of having a CYP2D6 non-NM predicted phenotype was highest in Algeria (61\%) and lowest in Gambia (2.7\%) while the probability for CYP2C19 was highest in India (80\%) and lowest in countries in the Americas, particularly Mexico (32\%). The mean total probability estimates of having a non-NM predicted phenotype worldwide were $36.4 \%$ and $61.9 \%$ for CYP2D6 and CYP2C19, respectively. We provide detailed tables and world maps summarizing clinically relevant data regarding the prevalence of CYP2D6 and CYP2C19 predicted phenotypes and demonstrating large inter-ethnic differences. Based on the documented probability estimates, pre-emptive pharmacogenetic testing is encouraged for every patient who will undergo therapy with a drug(s) that is metabolized by CYP2D6 and/or CYP2C19 pathways and should be considered in case of treatment resistance or serious side effects.
\end{abstract}

\section{Introduction}

Migration is a growing global phenomenon so that Western-trained psychiatrists are increasingly treating migrants with different cultural and ethnic backgrounds ${ }^{1}$. In the psychopharmacological treatment of migrants, variation in drug metabolism is an important aspect that must be taken into account ${ }^{2}$. In psychiatry, CYP2D6 and CYP2C19 are important drug-metabolizing enzymes $^{3-8}$. For example, drugs that are metabolized by CYP2D6 include first- and second-generation antipsychotics, selective serotonin receptor inhibitors, and tricyclic antidepressants ${ }^{6}$. Among those metabolized by CYP2C19 are benzodiazepines, selective serotonin

\footnotetext{
Correspondence: Anne B. Koopmans (koopmansanne@gmail.com)

${ }^{1}$ Parnassia Academy, Parnassia Psychiatric Institute, The Hague, the Netherlands ${ }^{2}$ School for Mental Health and Neuroscience, Maastricht University, Maastricht, the Netherlands

Full list of author information is available at the end of the article
}

receptor inhibitors, and tricyclic antidepressants ${ }^{6}$. Individuals' genetic variation is the most important factor influencing the kinetics of drug metabolism and thus may contribute to intolerability-related discontinuation or treatment failure?

The outcome of a pharmacogenetic test (i.e., a patient's genotype, sometimes also referred to as diplotype) can be translated into a predicted phenotype. A combination of functional and non-functional alleles is responsible for the activity of the enzymes. There are four phenotype groups: poor (PM), intermediate (IM), normal (NM) (previously referred to as "extensive"), and ultrarapid metabolizers (UM), which are used to predict whether and how well a drug is metabolized. The same drug dosage may lead to a higher plasma level in PMs and IMs, compared to NMs, because of slower drug clearance, while UMs may have lower plasma levels than NMs because of a higher rate of drug clearance. Plasma levels are often related to the 
efficacy of a drug and the risk of dose-related side effects, with more severe side effects found in PMs and IMs than in $\mathrm{NMs}^{10-15}$.

The prevalence of $C Y P$ polymorphisms also varies considerably across ethnic groups and plays a major role in inter-individual and inter-ethnic differences in drug metabolism and response ${ }^{16}$. For example, in European populations, just $2-3 \%$ of the population have a CYP2D6 UM profile, compared to 20-29\% in East-African populations $^{17,18}$. In contrast, CYP2C19 PMs are considerably more frequent in Asians $(\sim 12 \%)$ than in Europeans $(\sim 2 \%)^{19}$. Some allelic variants such as CYP2D6"40 and *45 are only seen in specific populations ${ }^{20,21}$.

The Clinical Pharmacogenetics Implementation Consortium (CPIC) has published guidelines with recommendations for drug choice and dosage based on phenotype predictions ${ }^{22-25}$.

Other groups, including the Royal Dutch Association for the Advancement of Pharmacy-Pharmacogenetics Working Group, have also published guidelines (information for both organizations is available through the Pharmacogenomics Knowledge Base at https://www.pharmgkb.org/ guidelines). CYP2D6 and CYP2C19 allele frequency information has been compiled by CPIC and is available at https://www.pharmgkb.org/page/cyp2d6RefMaterials. Gaedigk et al., Fricke-Galindo et al., and Llerena et al. have described CYP2D6 and CYP2C19 phenotype prediction from genotype across world populations ${ }^{17,19,26}$.

Although there is a wealth of information, pharmacogenetics is still not being widely used in clinical practice. Several studies have shown the relationship between CYP activity, blood serum levels, and side effects, but there have been few studies on clinical effectivity. Most of the studies are cross-sectional and observational, while prospective studies are often underpowered ${ }^{4,27-29}$. For some drugs, clinicians are used to working with therapeutic drug monitoring and they may prefer this over genotyping.

Another reason is the lack of education of practitioners on this topic and the belief that pharmacogenetics "is not ready" for use in daily clinical practice ${ }^{30-33}$. Despite these barriers, pharmacogenetics is increasingly being adopted by major health centers, and the body of literature in support of pharmacogenetic testing is growing $27,29,34-39$.

The aim of our meta-analysis was to assess studies reporting clinically useful information about $C Y P 2 D 6$ and CYP2C19 genotype frequencies across populations and ethnic groups worldwide. We introduce the concept of the non-normal metabolizer (non-NM) probability estimate, for which we calculated the sum-prevalence of a population for having a poor, intermediate, or ultrarapid CYP2D6 or CYP2C19 predicted phenotype. The sumprevalence of these three predicted phenotypes presents a single measurement for non-normal metabolism in the populations of interest. It is defined as the equivalent of the prevalence of $\mathrm{PM}+\mathrm{IM}+\mathrm{UM}$ predicted phenotypes of the enzyme in percentages.

\section{Methods}

For this study, we followed the checklist in the Preferred Reporting Items for Systematic Reviews and Meta-Analyses statement ${ }^{40,41}$. The protocol for the current systematic review was not registered prior to the review.

\section{Review of literature}

We conducted a literature review using the Embase, PubMed, Web of Science, and PsycINFO databases (1990-2019). The terms "CYP2D6" AND/OR "CYP2C19" AND "prevalence" OR "ethnicity" OR "race" AND "healthy subject" OR "normal control," and variations on these terms and the names of different countries and continents were used in all fields. The last search was conducted on July 3, 2019.

Our inclusion criteria were: (1) CYP2D6 or CYP2C19 allele frequencies from original data were reported; (2) the evaluated subjects did not have a specific disease (controls from case-control studies were included); (3) ethnicity was reported; (4) the article was published in English; (5) a minimum of 20 participants was investigated. If only an abstract was available, the article was included if all the above information was available; and (6) in order to be able to calculate a probability estimate, it was necessary to assess a minimum number of non-functional alleles and alleles with decreased function as well as a number of gene duplications. The genotyping assay included at least the following allelic variants: CYP2C19*2 and *17 in Africans, Americans, Europeans, Middle Easterners, Central/South East-Asians and African Americans; CYP2C19*2, *3, and *17 in Oceanians and CYP2C19*2 and *3 in East Asians; CYP2D6*2, *5, *17, *29, and *41 in Africans; CYP2D6*2 and *4 in Americans; CYP2D6*2 and "10 in East Asians; CYP2D6*2, "4, and "41 in Europeans; CYP2D6*2, *4, "10, and *41 in Middle Easterners and Central/South East-Asians; CYP2D6*5 in Oceanians; and CYP2D6*2, *4, *17, and *29 in African Americans.

An eligibility assessment was performed independently in a standardized manner by A.B.K. and D.J.V. The first screening was based on the article abstracts; the next selection was based on the full text. Disagreements between the two reviewers were resolved by discussion to reach a consensus.

\section{Data extraction}

The data were independently extracted from the studies by two investigators (A.B.K. and P.B.) and randomly checked by two investigators (D.J.V. and A.B.K.). For some studies, authors were contacted for clarification of the data.

Information was extracted from each study as follows: (1) ethnicity of the participants; (2) definition of ethnicity 
(self-reported or genetic ancestry); (3) country of the studied population; (4) number of study participants; (5) study design (prevalence study, case-control study, experimental study); (6) allele frequencies; (7) diplotype frequencies; (8) predicted phenotype frequencies.

Star (") alleles were assigned according to the Pharmacogenetic Variation (PharmVar) Consortium at PharmVar.org ${ }^{42,43}$.

\section{Ethnicity and geographical regions}

To be able to compare outcomes with previous metaanalyses, reported ethnicity was assigned to geographic regions, as done in previous meta-analysis about this subject, according to the Human Genome Diversity Project ${ }^{44}$. Seven major regions were considered: Africa, Americas (including Latino Americans and indigenous inhabitants of North America and Canada), East Asia, Europe (including North Americans and Canadians), the Middle East, Oceania, and Central/Southeast Asia; with one exception, namely, that African Americans were listed separately from Africans ${ }^{17}$. Here the frequencies of PM, IM, normal metabolizer (NM), and UM are reported by ethnicity, whereas the probability estimates of being a non-NM are reported by country. In many studies, these two factorscountry and ethnicity-overlap, but for some studies we had to assign an ethnicity to a country to be able to show the information in world maps (i.e., the two factors were not distinguished). The origin of the investigated ethnicity determined the country and region to which a population was assigned. An exception was made for Latin America, in which the population is an admixture of multiple origins (e.g., European, African, Asian, and Amerindian) and no clear lineages can be determined; they were all considered as populations of the Americas and determined as belonging to the country they live in ${ }^{45,46}$. For some ethnicities, we could not determine a country of origin (for example, East Asians or Europeans) so we have indicated them as "missing" in the figures.

\section{Translation of genotype into phenotype}

For each geographical region, the mean frequency of alleles was determined. In order to predict CYP2D6 and CYP2C19 phenotype frequencies from genotype data, we applied the activity score (AS) system to both genes (the AS system is widely used for CYP2D6 and was adapted to CYP2C19 to facilitate the translation process) ${ }^{47}$. Briefly, a normal function allele was valued as 1 , decreased function alleles as 0.25 or 0.5 , a non-functional allele as 0 , and increased function allele as 1.5. Gene duplications received double the value of their singleton counterparts. Homozygous carriers of non-functional alleles were classified as PMs $(\mathrm{AS}=0)$. Carriers with one functional or decreased function allele and one non-functional allele and those carrying two decreased function alleles were
Table 1 Functionality of CYP2D6 and CYP2C19 alleles (https://www.pharmvar.org/gene).

\begin{tabular}{|c|c|c|}
\hline & CYP2D6 & CYP2C19 \\
\hline 0 & $\begin{array}{l}* 3-{ }^{*} 8, * 15, * 18, * 31, * 36, * 47, * 51, \\
* 56, * 57, * 62, * 92, * 100, \text { and }{ }^{*} 101\end{array}$ & $\begin{array}{l}{ }^{*} 2, *^{*}, * 4, * 5, * 6, * 7, * 8, \\
*^{*} 23 \text {, and }{ }^{*} 24\end{array}$ \\
\hline $0.25-0.5$ & $\begin{array}{l}* 9, * 10, * 17, * 29, * 41, * 49, * 50, * 54, \\
* 55, * 59 \text {, and } * 72\end{array}$ & $\begin{array}{l}{ }^{*} 9, * 10, * 12, * 16, * 25 \\
\text { and }{ }^{*} 27\end{array}$ \\
\hline 1 & $* 1, * 2, * 27, * 39, * 45, * 46$, and $* 48$ & $* 1, * 13, * 15$ and $* 18$ \\
\hline 1.5 & $*_{53}$ & $*_{17}$ \\
\hline Unknown & $* 43, * 60, * 65, * 82, * 84, * 85$, and $* 86$ & \\
\hline
\end{tabular}

classified as IMs (AS $=0.25-1)^{47}$. Homozygous carriers of normal function alleles and heterozygous carriers with one decreased function and one normal function allele were classified as NMs (AS $=1.25-2.25)^{47}$. Carriers of one or more increased function alleles and carriers of a duplication or multiplication of a functional allele were classified as UMs $(\mathrm{AS}>2.25)^{47} \quad$ (https://cpicpgx.org/ resources/term-standardization/). CYP2C19 rapid and ultrarapid metabolizers were pooled and analyzed as UMs. The functionality of the CYP2D6 and CYP2C19 alleles was classified as listed by PharmVar in Table 1.

In this meta-analysis, we applied strict criteria. To maximize the accuracy of the frequencies of the predicted phenotypes, we only predicted a phenotype if the original publication reported a minimum of non-/decreased function alleles and the assays included tests for gene duplications. Since the prevalence of alleles differed greatly per region, we used criteria specific for each geographical region. Alleles more prevalent than $0.05(5 \%)$ in the major region (Table 2) had to be investigated in the countries within that region to be included in the phenotype predictions.

\section{Calculations and statistics}

All analyses were performed with IBM SPSS Statistics Version 25. If only diplotypes were reported, single allele frequencies were calculated. If only single allele frequencies were reported, diplotype frequencies were calculated using the Hardy-Weinberg equilibrium $\left(p^{2}+2 p q+q^{2}=1\right)$. For studies that did not report the prevalence of $C Y P 2 D 6^{*} 1$, the allele frequency was calculated as $100 \%$ minus the sum of variants ${ }^{17}$. The diplotypes were translated into predicted phenotypes according to the CPIC.

We introduce here the concept of the non-NM probability estimate. It is defined as the sum of the prevalence of PM+ $\mathrm{IM}+\mathrm{UM}$ predicted phenotypes of the enzymes CYP2D6 or CYP2C19 in percentages. Thus it is equivalent to the prevalence (as percentage) of all the non-normal phenotypes in a population. We use the term probability estimate exclusively in this sense and it is in fact a proportion of the 


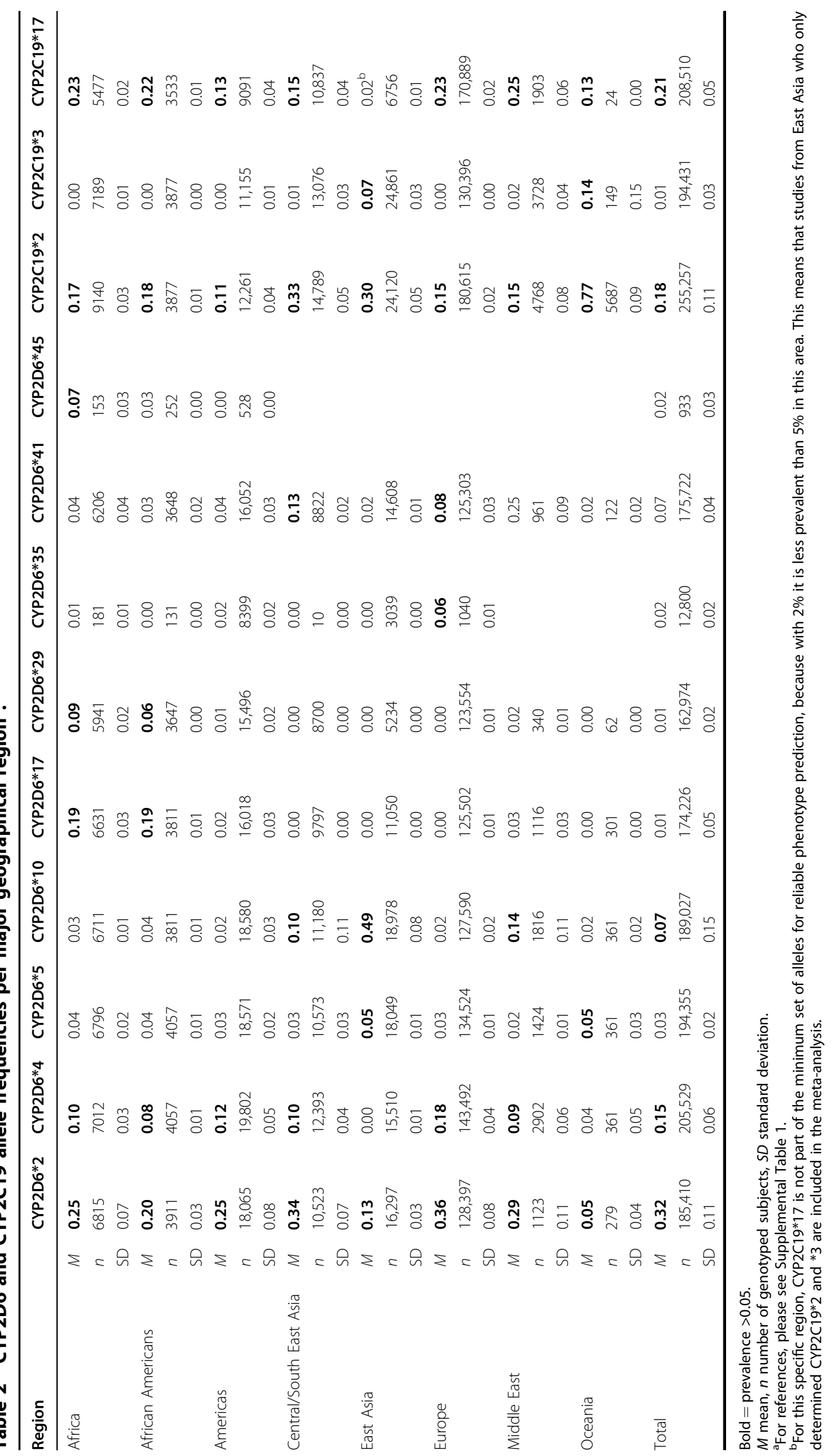




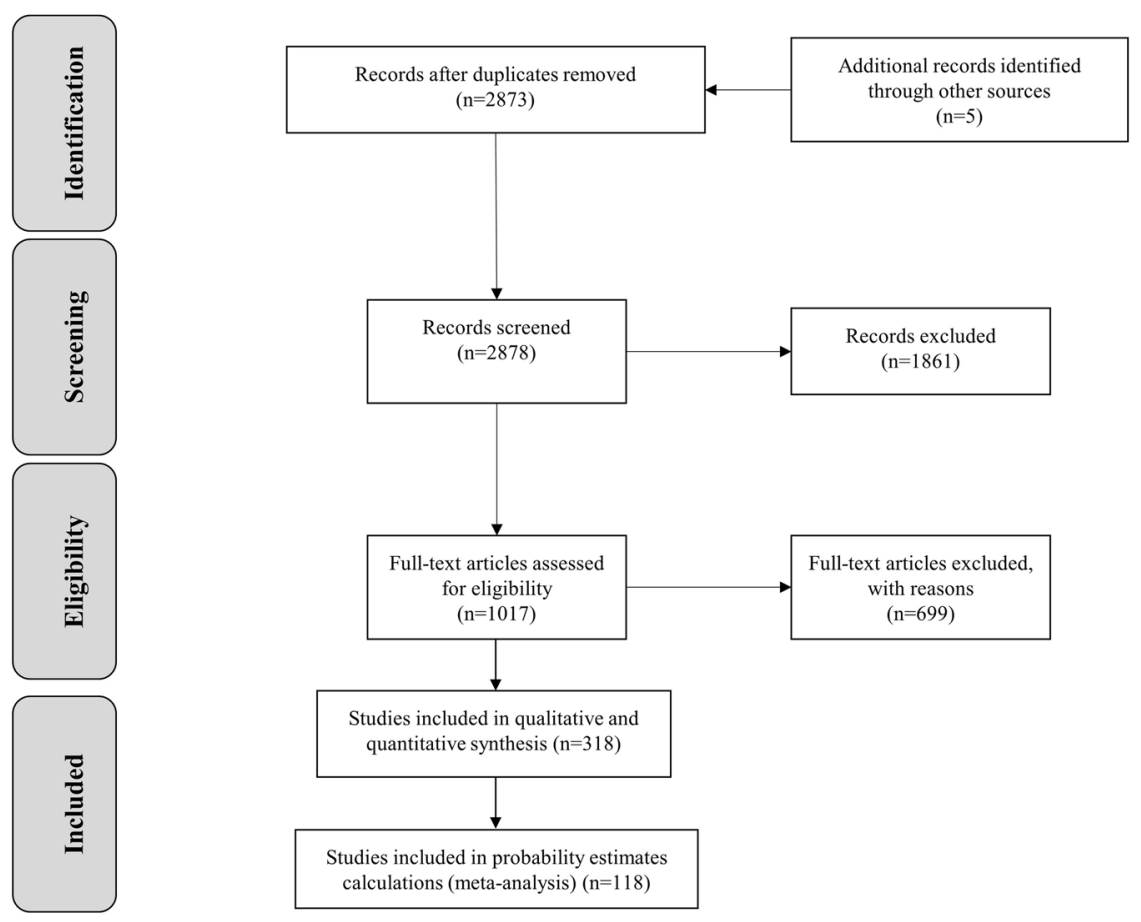

Fig. 1 PRISMA flow diagram of the studies included in the analyses. $n=$ number of studies.

possible outcomes in a population. It is equal to $100 \%$ minus the percentage of NM in a given population.

The studies were weighted by sample size (number of participants) when we calculated the mean predicted phenotypes per country and ethnicity.

\section{Results}

Of the 2873 publications retrieved from the database, 318 original research papers met our inclusion criteria (Fig. 1). The analyses of CYP2D6 ( $n=200$ papers) and CYP2C19 ( $n=159$ papers) included 261,296 and 257,745 healthy individuals. The alleles most frequently investigated were $C Y P 2 D 6 * 1-{ }^{*} 6, * 10, * 17$, and $C Y P 2 C 19 * 1-* 3$. Allele frequencies are shown per major geographical region in Table 2 (for references, please see Supplemental Table 1). The most frequently observed variant alleles across all subjects were $C Y P 2 D 6 * 2, * 4, * 10$, and *41 and CYP2C19*2 and *17. As expected, allele frequencies varied substantially among ethnicities and countries. We found 89 studies that reported on more than one ethnic group. Overall, African and Middle Eastern countries were underrepresented, while European populations were the most frequently investigated.

\section{CYP2D6}

\section{Prevalence of predicted phenotypes by ethnicity}

Predicted phenotype was reported or could be inferred from 51 studies for 116 ethnicities, covering $n=194,714$ individuals. These studies were selected for fulfilling the minimum number of alleles tested as prescribed by our region-specific criteria. Due to the high frequency of allele duplications, high percentages of CYP2D6 UM were found in the Mozabite people, a Berber ethnic group in the Sahara, North Africa $(39.5 \%)^{48}$, in non-Austronesian Melanesians $(21.5 \%)^{48}$, and in the ethno-religious Druze from the Middle East $(21.4 \%)^{48}$. High percentages of CYP2D6 PM were found in Europeans, for example, in the British $(12.1 \%)^{49}$, the Danish $(10.6 \%)^{50}$, and Basque (French) people $(9.7 \%)^{48}$ due to the high frequency of CYP2D6*4. Frequencies of CYP2D6 predicted phenotypes by ethnicity are summarized in Table 3 (for references, please see Supplemental Table 2).

\section{Probability estimates by country}

The probability of having a CYP2D6 non-NM predicted phenotype is the highest in Algeria (non-NM probability estimated to be $61.2 \%$; $4, * 17, * 41$, and duplications $)^{48}$, Argentina (non-NM probability estimate $51.4 \%$; *4, *41, and duplications) ${ }^{51}$, and France (non-NM probability estimate $50.4 \% ; * 4 * 5, * 41$, and duplications) ${ }^{48}$. The CYP2D6 non-NM probability estimate was lowest in several populations from Africa (Gambia 2.7\%, Kenya 4.0\%, and Sierra Leone 5.9\%) and South-East Asia (Vietnam 5.1\%, Sri Lanka 7.8\%) ${ }^{49}$. See Fig. 2 for CYP2D6 nonNM probability estimates and Fig. 3 for CYP2D6 non-NM probability estimates plotted on a world map. 
Table 3 Mean frequencies of CYP2D6 predicted phenotypes per ethnicity ${ }^{\mathrm{a}}$.

\begin{tabular}{|c|c|c|c|c|c|c|c|c|c|c|c|c|}
\hline \multirow[t]{2}{*}{ Ethnicity } & \multirow[b]{2}{*}{$\mathbf{n}$} & \multicolumn{3}{|c|}{ PM } & & & & & & & & Others $^{\mathrm{b}}$ \\
\hline & & & $\mathbf{M}$ & SD & M & SD & $\mathbf{M}$ & SD & & M & SD & M \\
\hline Africa & & & & & & & & & & & & \\
\hline African & 5203 & - & 0.028 & 0.000 & 0.394 & 0.000 & 0.528 & 0.000 & E. & 0.059 & 0.000 & 0.000 \\
\hline African (North and East) & 65 & $\mathrm{~F}$ & 0.017 & 0.000 & 0.195 & 0.000 & 0.346 & 0.000 & 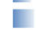 & 0.069 & 0.000 & 0.373 \\
\hline Biaka, Pygmies (Subsaharan Africa) & 36 & 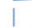 & 0.002 & 0.000 & 0.141 & 0.000 & 0.802 & 0.000 & & 0.000 & 0.000 & 0.055 \\
\hline Colored (South African) & 199 & 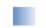 & 0.020 & 0.010 & 0.440 & 0.010 & 0.492 & 0.002 & I & 0.017 & 0.009 & 0.031 \\
\hline Esan (Nigerian) & 99 & 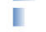 & 0.010 & 0.000 & 0.111 & 0.000 & 0.798 & 0.000 & & 0.000 & 0.000 & 0.081 \\
\hline Gambian & 113 & I & 0.009 & 0.000 & 0.018 & 0.000 & 0.912 & 0.000 & & 0.000 & 0.000 & 0.061 \\
\hline Luhya in Webuye (Kenian) & 99 & & 0.000 & 0.000 & 0.040 & 0.000 & 0.848 & 0.000 & & 0.000 & 0.000 & 0.112 \\
\hline Mandenka (Subsaharan Africa) & 24 & r & 0.035 & 0.000 & 0.471 & 0.000 & 0.446 & 0.000 & & 0.000 & 0.000 & 0.047 \\
\hline Mende (Sierra Leonean) & 85 & 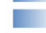 & 0.035 & 0.000 & 0.024 & 0.000 & 0.824 & 0.000 & & 0.000 & 0.000 & 0.117 \\
\hline Mozabite (Algerian) & 30 & - & 0.023 & 0.000 & 0.194 & 0.000 & 0.366 & 0.000 & $\square$ & 0.395 & 0.000 & 0.022 \\
\hline North African & 31 & 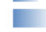 & 0.032 & 0.000 & 0.226 & 0.000 & 0.581 & 0.000 & 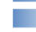 & 0.161 & 0.000 & 0.000 \\
\hline South African & 71 & I & 0.005 & 0.000 & 0.604 & 0.000 & 0.352 & 0.000 & I & 0.010 & 0.000 & 0.030 \\
\hline West African & 28 & & 0.000 & 0.000 & 0.322 & 0.000 & 0.607 & 0.000 & 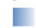 & 0.071 & 0.000 & 0.000 \\
\hline Xhosa (South African) & 53 & 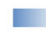 & 0.038 & 0.000 & 0.363 & 0.000 & 0.480 & 0.000 & 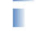 & 0.038 & 0.000 & 0.081 \\
\hline Yoruba (Nigerian) (Ibadan) & 108 & & 0.000 & 0.000 & 0.046 & 0.000 & 0.898 & 0.000 & & 0.000 & 0.000 & 0.056 \\
\hline Yoruba (Subsaharan Africa) & 25 & 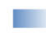 & 0.032 & 0.000 & 0.338 & 0.000 & 0.565 & 0.000 & $\mathbf{E}$ & 0.064 & 0.000 & 0.000 \\
\hline African American & 443 & $\mathbf{E}$ & 0.024 & 0.006 & 0.341 & 0.107 & 0.563 & 0.097 & I & 0.029 & 0.014 & 0.042 \\
\hline Americas & & & & & & & & & & & & \\
\hline Admixed Latin Americans & 8246 & 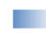 & 0.033 & 0.006 & 0.303 & 0.031 & 0.672 & 0.006 & I & 0.023 & 0.023 & 0.000 \\
\hline African Caribbean (Barbados) & 96 & 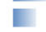 & 0.021 & 0.000 & $\quad 0.063$ & 0.000 & 0.854 & 0.000 & & 0.000 & 0.000 & 0.062 \\
\hline African Caribbean from Barbados & & I & & & & & & & 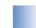 & & & \\
\hline (Costa Rica) & 103 & 1 & 0.012 & 0.000 & 0.267 & 0.000 & 0.546 & 0.000 & L & 0.079 & 0.000 & 0.095 \\
\hline Afro-Latin Americans & 93 & 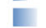 & 0.022 & 0.000 & 0.258 & 0.000 & 0.645 & 0.000 & 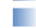 & 0.075 & 0.000 & 0.000 \\
\hline American Indian & 187 & E & 0.059 & 0.000 & 0.032 & 0.000 & 0.872 & 0.000 & | & 0.011 & 0.000 & 0.027 \\
\hline Brazilian (North East) & 261 & 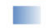 & 0.025 & 0.000 & 0.281 & 0.000 & 0.627 & 0.000 & I & 0.019 & 0.000 & 0.047 \\
\hline Brazilian (North) & 246 & 2 & 0.021 & 0.000 & 0.253 & 0.000 & 0.649 & 0.000 & i & 0.026 & 0.000 & 0.051 \\
\hline Brazilian (South East) & 258 & 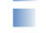 & 0.020 & 0.000 & 0.271 & 0.000 & 0.640 & 0.000 & i & 0.023 & 0.000 & 0.045 \\
\hline Brazilian (South) & 255 & E & 0.024 & 0.000 & 0.281 & 0.000 & 0.632 & 0.000 & i & 0.013 & 0.000 & 0.049 \\
\hline Brazilian from African descent & 92 & 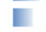 & 0.017 & 0.000 & 0.320 & 0.000 & 0.625 & 0.000 & I & 0.029 & 0.000 & 0.008 \\
\hline Brazilian from European descent & 87 & 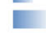 & 0.030 & 0.000 & 0.288 & 0.000 & 0.585 & 0.000 & 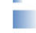 & 0.077 & 0.000 & 0.020 \\
\hline Central Native Americans & 196 & 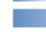 & 0.102 & 0.000 & 0.342 & 0.000 & 0.520 & 0.000 & 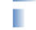 & 0.036 & 0.000 & 0.000 \\
\hline Colombian & 215 & 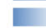 & 0.037 & 0.005 & 0.219 & 0.156 & 0.674 & 0.100 & i & 0.010 & 0.008 & 0.060 \\
\hline Coras (Mexican) & 81 & & 0.000 & 0.000 & I $\quad 0.049$ & 0.000 & 0.864 & 0.000 & 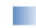 & 0.086 & 0.000 & 0.000 \\
\hline Cuban from European descent & 130 & $\mathbf{E}$ & 0.023 & 0.000 & 0.292 & 0.000 & 0.623 & 0.000 & 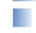 & 0.062 & 0.000 & 0.000 \\
\hline Dutch Caribbean & 332 & 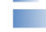 & 0.043 & 0.000 & 0.325 & 0.000 & 0.613 & 0.000 & I & 0.026 & 0.000 & 0.000 \\
\hline Ecuadorian & 257 & 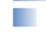 & 0.021 & 0.012 & 0.205 & 0.044 & 0.730 & 0.004 & I & 0.034 & 0.024 & 0.009 \\
\hline Huicholes (Mexican) & 107 & & 0.000 & 0.000 & 0.150 & 0.000 & 0.645 & 0.000 & 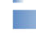 & 0.206 & 0.000 & 0.000 \\
\hline Iberians & 1530 & 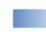 & 0.047 & 0.000 & 0.341 & 0.000 & 0.563 & 0.000 & 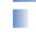 & 0.050 & 0.000 & 0.000 \\
\hline Indo-Trinidad & 167 & E & 0.025 & 0.000 & 0.298 & 0.000 & 0.622 & 0.000 & I & 0.025 & 0.000 & 0.031 \\
\hline Karitiana (South American) & 24 & & 0.000 & 0.000 & 0.000 & 0.000 & 0.837 & 0.000 & 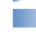 & 0.159 & 0.000 & 0.004 \\
\hline Lacandones (Mexican) & 154 & & 0.000 & 0.000 & 0.214 & 0.000 & 0.758 & 0.000 & I & 0.025 & 0.000 & 0.003 \\
\hline Maya (Mexican) & 44 & & 0.000 & 0.000 & 0.205 & 0.000 & 0.591 & 0.000 & F & 0.182 & 0.000 & 0.023 \\
\hline Maya (South American) & 25 & I & 0.004 & 0.000 & 0.106 & 0.000 & 0.781 & 0.000 & 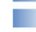 & 0.109 & 0.000 & 0.000 \\
\hline Mestizo (Cuban) & 126 & 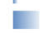 & 0.024 & 0.000 & 0.268 & 0.000 & 0.654 & 0.000 & 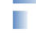 & 0.047 & 0.000 & 0.007 \\
\hline Mestizo (Mexican) & 439 & 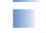 & 0.019 & 0.005 & 0.255 & 0.012 & 0.663 & 0.033 & 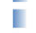 & 0.043 & 0.022 & 0.020 \\
\hline Mestizo (Nicarguan) & 235 & 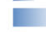 & 0.038 & 0.002 & 0.332 & 0.040 & 0.604 & 0.032 & I & 0.022 & 0.008 & 0.004 \\
\hline Mexican & 1081 & E & 0.012 & 0.011 & 0.157 & 0.075 & 0.765 & 0.042 & E & 0.054 & 0.056 & 0.011 \\
\hline North Native Americans & 956 & I & 0.003 & 0.000 & 0.129 & 0.000 & 0.773 & 0.000 & 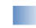 & 0.095 & 0.000 & 0.000 \\
\hline Peruvian & 85 & & 0.000 & 0.000 & 0.000 & 0.000 & 0.918 & 0.000 & & 0.000 & 0.000 & 0.082 \\
\hline Pima (South American) & 25 & I & 0.006 & 0.000 & 0.134 & 0.000 & 0.718 & 0.000 & 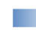 & 0.141 & 0.000 & 0.000 \\
\hline Puerto Rican & 104 & 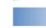 & 0.058 & 0.000 & 0.048 & 0.000 & 0.779 & 0.000 & & 0.000 & 0.000 & 0.115 \\
\hline South American & 25 & 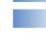 & 0.040 & 0.000 & 0.200 & 0.000 & 0.720 & 0.000 & I & 0.040 & 0.000 & 0.000 \\
\hline South Native Americans & 214 & & 0.000 & 0.000 & 0.126 & 0.000 & 0.869 & 0.000 & 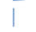 & 0.005 & 0.000 & 0.000 \\
\hline Surui (South American) & 21 & & 0.000 & 0.000 & 0.000 & 0.000 & 1.000 & 0.000 & & 0.000 & 0.000 & 0.000 \\
\hline Tarahumares (Mexican) & 74 & E & 0.014 & 0.000 & 0.257 & 0.000 & 0.622 & 0.000 & 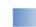 & 0.122 & 0.000 & 0.000 \\
\hline Tepehuanos (Mexican) & 129 & & 0.000 & 0.000 & 0.015 & 0.000 & 0.931 & 0.000 & 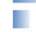 & 0.054 & 0.000 & 0.000 \\
\hline White Latin Americans & 282 & 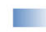 & 0.032 & 0.000 & 0.302 & 0.000 & 0.599 & 0.000 & 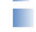 & 0.067 & 0.000 & 0.000 \\
\hline Central/South East Asia & & & & & & & & & & & & \\
\hline Balochi (Central/South Asia) & 25 & I & 0.010 & 0.000 & 0.216 & 0.000 & 0.738 & 0.000 & I & 0.036 & 0.000 & 0.000 \\
\hline Bengali & 86 & 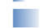 & 0.023 & 0.000 & 0.058 & 0.000 & 0.884 & 0.000 & & 0.000 & 0.000 & 0.035 \\
\hline Brahui (Central/South Asia) & 25 & I & 0.010 & 0.000 & 0.206 & 0.000 & 0.784 & 0.000 & & 0.000 & 0.000 & 0.000 \\
\hline Burusho (Central/South Asia) & 25 & I & 0.010 & 0.000 & 0.190 & 0.000 & 0.800 & 0.000 & & 0.000 & 0.000 & 0.000 \\
\hline Gujarati (Indian) & 103 & [ & 0.010 & 0.000 & 0.029 & 0.000 & 0.951 & 0.000 & & 0.000 & 0.000 & 0.010 \\
\hline Hazara (Central/South Asia) & 25 & 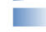 & 0.032 & 0.000 & 0.295 & 0.000 & 0.608 & 0.000 & E. & 0.064 & 0.000 & 0.000 \\
\hline Kalash (Central/South Asia) & 25 & I & 0.006 & 0.000 & 0.151 & 0.000 & 0.843 & 0.000 & & 0.000 & 0.000 & 0.000 \\
\hline Makrani (Central/South Asia) & 25 & 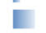 & 0.020 & 0.000 & 0.260 & 0.000 & 0.720 & 0.000 & & 0.000 & 0.000 & 0.000 \\
\hline Pathan (Central/South Asia) & 25 & [ & 0.010 & 0.000 & 0.202 & 0.000 & 0.753 & 0.000 & I & 0.036 & 0.000 & 0.000 \\
\hline Punjabi (Indian) & 96 & I & 0.010 & 0.000 & $\quad 0.073$ & 0.000 & 0.906 & 0.000 & & 0.000 & 0.000 & 0.011 \\
\hline Sindhi (Central/South Asia) & 25 & $=$ & 0.026 & 0.000 & 0.309 & 0.000 & 0.666 & 0.000 & & 0.000 & 0.000 & 0.000 \\
\hline South Asian & 8283 & 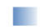 & 0.021 & 0.001 & 0.298 & 0.004 & 0.733 & 0.003 & & 0.000 & 0.000 & 0.000 \\
\hline South East Asia & 32 & & 0.000 & 0.000 & 0.281 & 0.000 & 0.719 & 0.000 & & 0.000 & 0.000 & 0.000 \\
\hline Tamil (Sri Lankan) & 102 & 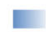 & 0.029 & 0.000 & $\quad 0.039$ & 0.000 & 0.882 & 0.000 & I & 0.010 & 0.000 & 0.040 \\
\hline Telugu (Indian) & 102 & 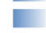 & 0.029 & 0.000 & 0.049 & 0.000 & 0.882 & 0.000 & i & 0.010 & 0.000 & 0.030 \\
\hline Uyghurs (Central/South Asia) & 25 & I & 0.010 & 0.000 & 0.220 & 0.000 & 0.770 & 0.000 & & 0.000 & 0.000 & 0.000 \\
\hline Viet Kinh (Vietnamese) & 99 & & 0.000 & 0.000 & 0.051 & 0.000 & 0.949 & 0.000 & & 0.000 & 0.000 & 0.001 \\
\hline Western India & 150 & E & 0.016 & 0.000 & 0.251 & 0.000 & 0.694 & 0.000 & I & 0.038 & 0.000 & 0.000 \\
\hline
\end{tabular}


Table 3 continued

\begin{tabular}{|c|c|c|c|c|c|c|c|c|c|c|c|c|c|}
\hline \multicolumn{14}{|l|}{ East Asia } \\
\hline Chinese & 715 & E & 0.021 & 0.018 & 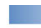 & 0.409 & 0.012 & 0.559 & 0.033 & I & 0.014 & 0.007 & 0.000 \\
\hline Dai (Chinese) & 93 & & 0.000 & 0.000 & I & 0.032 & 0.000 & 0.957 & 0.000 & & 0.000 & 0.000 & 0.011 \\
\hline East Asian & 4327 & I & 0.007 & 0.000 & 口 & 0.536 & 0.000 & 0.451 & 0.000 & I & 0.022 & 0.000 & 0.000 \\
\hline Han (Chinese) & 620 & I & 0.008 & 0.007 & 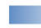 & 0.293 & 0.192 & 0.660 & 0.219 & | & 0.007 & 0.009 & 0.031 \\
\hline Japanese & 878 & I & 0.005 & 0.003 & - & 0.253 & 0.095 & 0.732 & 0.102 & I & 0.012 & 0.007 & 0.000 \\
\hline Shanghai (Chinese) & 100 & & 0.000 & 0.000 & $\mathbf{E}$ & 0.395 & 0.000 & 0.582 & 0.000 & I & 0.012 & 0.000 & 0.011 \\
\hline Shantou (Chinese) & 100 & E & 0.013 & 0.000 & E & 0.564 & 0.000 & 0.410 & 0.000 & I & 0.013 & 0.000 & 0.000 \\
\hline Shenyang (Chinese) & 100 & 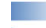 & 0.045 & 0.000 & 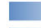 & 0.337 & 0.000 & 0.584 & 0.000 & I & 0.022 & 0.000 & 0.011 \\
\hline Tibetan Chinese & 96 & & 0.000 & 0.000 & 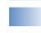 & 0.156 & 0.000 & 0.844 & 0.000 & & 0.000 & 0.000 & 0.000 \\
\hline Xi'an (Chinese) & 100 & E & 0.022 & 0.000 & $\mathbf{E}$ & 0.381 & 0.000 & 0.544 & 0.000 & I & 0.033 & 0.000 & 0.022 \\
\hline Yakut (East Asia) & 25 & E & 0.014 & 0.000 & $\mathbf{E}$ & 0.221 & 0.000 & 0.696 & 0.000 & E & 0.069 & 0.000 & 0.000 \\
\hline \multicolumn{14}{|l|}{ Europe } \\
\hline Basque (French) & 24 & - & 0.097 & 0.000 & $\mathbf{\square}$ & 0.445 & 0.000 & 0.431 & 0.000 & I & 0.028 & 0.000 & 0.000 \\
\hline British & 91 & 口 & 0.121 & 0.000 & 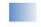 & 0.121 & 0.000 & 0.747 & 0.000 & & 0.000 & 0.000 & 0.011 \\
\hline Danish & 122 & 口 & 0.106 & 0.000 & - & 0.352 & 0.000 & 0.525 & 0.000 & 1 & 0.008 & 0.000 & 0.009 \\
\hline Estonian & 44000 & 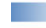 & 0.050 & 0.000 & I & 0.039 & 0.000 & 0.888 & 0.000 & I & 0.024 & 0.000 & 0.000 \\
\hline Finnish & 956 & 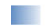 & 0.026 & 0.008 & I & 0.030 & 0.000 & 0.877 & 0.011 & $E$ & 0.065 & 0.022 & 0.050 \\
\hline Finnish (East) & 86 & E & 0.021 & 0.000 & 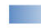 & 0.241 & 0.000 & 0.670 & 0.000 & E & 0.068 & 0.000 & 0.000 \\
\hline Finnish (West) & 56 & 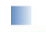 & 0.018 & 0.000 & $\mathbf{E}$ & 0.220 & 0.000 & 0.672 & 0.000 & 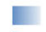 & 0.091 & 0.000 & 0.000 \\
\hline French & 25 & 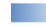 & 0.048 & 0.000 & $\mathbf{E}$ & 0.360 & 0.000 & 0.561 & 0.000 & I & 0.031 & 0.000 & 0.000 \\
\hline German & 113 & 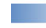 & 0.070 & 0.000 & E & 0.350 & 0.000 & 0.540 & 0.000 & I & 0.040 & 0.000 & 0.000 \\
\hline Hungarian & 112 & 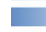 & 0.083 & 0.000 & $\bar{E}$ & 0.365 & 0.000 & 0.508 & 0.000 & I & 0.019 & 0.000 & 0.024 \\
\hline Iberian (Spanish) & 107 & 1 & 0.028 & 0.000 & I & 0.019 & 0.000 & 0.916 & 0.000 & & 0.000 & 0.000 & 0.037 \\
\hline Jewish (Ashkenazi) & 5249 & 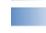 & 0.043 & 0.002 & - & 0.371 & 0.004 & 0.502 & 0.003 & $\mathbb{E}$ & 0.087 & 0.005 & 0.000 \\
\hline Lithuania & 104 & [ & 0.012 & 0.000 & 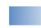 & 0.207 & 0.000 & 0.781 & 0.000 & & 0.000 & 0.000 & 0.000 \\
\hline Ravenna Italian & 122 & 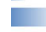 & 0.040 & 0.000 & 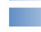 & 0.319 & 0.000 & 0.543 & 0.000 & 1 & 0.006 & 0.000 & 0.092 \\
\hline Roma (Italian) & 122 & 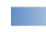 & 0.066 & 0.000 & 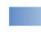 & 0.312 & 0.000 & 0.610 & 0.000 & I & 0.008 & 0.000 & 0.004 \\
\hline Russian & 25 & 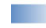 & 0.040 & 0.000 & 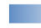 & 0.302 & 0.000 & 0.557 & 0.000 & 1 & 0.061 & 0.000 & 0.040 \\
\hline Sardinian (Italian) & 28 & 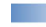 & 0.063 & 0.000 & 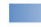 & 0.368 & 0.000 & 0.519 & 0.000 & I & 0.026 & 0.000 & 0.024 \\
\hline Spanish & 805 & 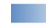 & 0.054 & 0.000 & $\mathbf{E}$ & 0.351 & 0.000 & 0.539 & 0.000 & E & 0.053 & 0.000 & 0.002 \\
\hline Toscani (Italian) & 107 & E & 0.047 & 0.000 & 【 & 0.065 & 0.000 & 0.869 & 0.000 & & 0.000 & 0.000 & 0.019 \\
\hline \multicolumn{14}{|l|}{ Middle East } \\
\hline Arabian Bedouins (Israel) & 50 & E & 0.020 & 0.000 & 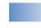 & 0.220 & 0.000 & 0.720 & 0.000 & I & 0.040 & 0.000 & 0.000 \\
\hline Bedouin (Middle Eastern) & 49 & I & 0.013 & 0.000 & 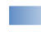 & 0.245 & 0.000 & 0.590 & 0.000 & E & 0.049 & 0.000 & 0.103 \\
\hline \multicolumn{14}{|l|}{ Oceania } \\
\hline Aboriginal (North West Australia) & 239 & I & 0.004 & 0.000 & $\mathbf{E}$ & 0.180 & 0.000 & 0.816 & 0.000 & & 0.000 & 0.000 & 0.000 \\
\hline non-Austronesian Melanesian & 22 & & 0.000 & 0.000 & 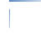 & 0.002 & 0.000 & 0.781 & 0.000 & - & 0.215 & 0.000 & 0.002 \\
\hline Total & 194714 & E & 0.049 & 0.018 & $\mathbf{E}$ & 0.287 & 0.149 & 0.644 & 0.154 & I & 0.028 & 0.019 & 0.000 \\
\hline
\end{tabular}

$M$ mean, $n$ number of genotyped subjects, $S D$ standard deviation, $P M$ poor metabolizers, $I M$ intermediate metabolizers, $N M$ normal metabolizers, UM ultrarapid metabolizers.

${ }^{\mathrm{a}}$ For references, please see Supplemental Table 2.

${ }^{b}$ An SNP combination that could not be assigned to a known allele/phenotype.

\section{CYP2C19}

\section{Prevalence of predicted phenotypes by ethnicity}

Data were retrieved from 73 studies describing 225,173 subjects of 85 ethnic groups/countries. These studies were selected for fulfilling the minimum number of alleles tested as prescribed by our region-specific criteria. The UM predicted phenotype is rather common in Ecuadorian Mestizos $(41.4 \%)^{52}$, Dargins $(39.8 \%)^{53}$, and ethnic groups living in the North Caucasus in Russia and in Burushu, Pakistan (39.0\%) ${ }^{54,55}$ owing to a high prevalence of $>20 \%$ of $C Y P 2 C 19 * 17$ in all these populations. High percentages of CYP2C19 PMs were found in Indian and Pakistani populations (Naik $31.0 \%{ }^{56}$ and Saraiki $20.0 \%{ }^{55}$ ),
Tohoku Japanese $(18.9 \%)^{57}$, and in Chinese Hui $(28.0 \%)^{58,59}$ due to the presence of the CYP2C19*2 and *3 non-functional alleles. Table 4 summarizes the frequencies of CYP2C19 predicted phenotypes by ethnicity (for references, please see Supplemental Table 3).

\section{Probability estimates by country}

The probability of having a CYP2C19 non-NM predicted phenotype due to high frequencies of the nonfunctional $C Y P 2 C 19 * 2$ allele and/or the increased function CYP2C19*17 allele is highest in India (non-NM probability estimate $80.1 \%)^{56,60,61}$, Pakistan (non-NM probability estimate $74.8 \%)^{55,62,63}$, and Iran (non-NM 


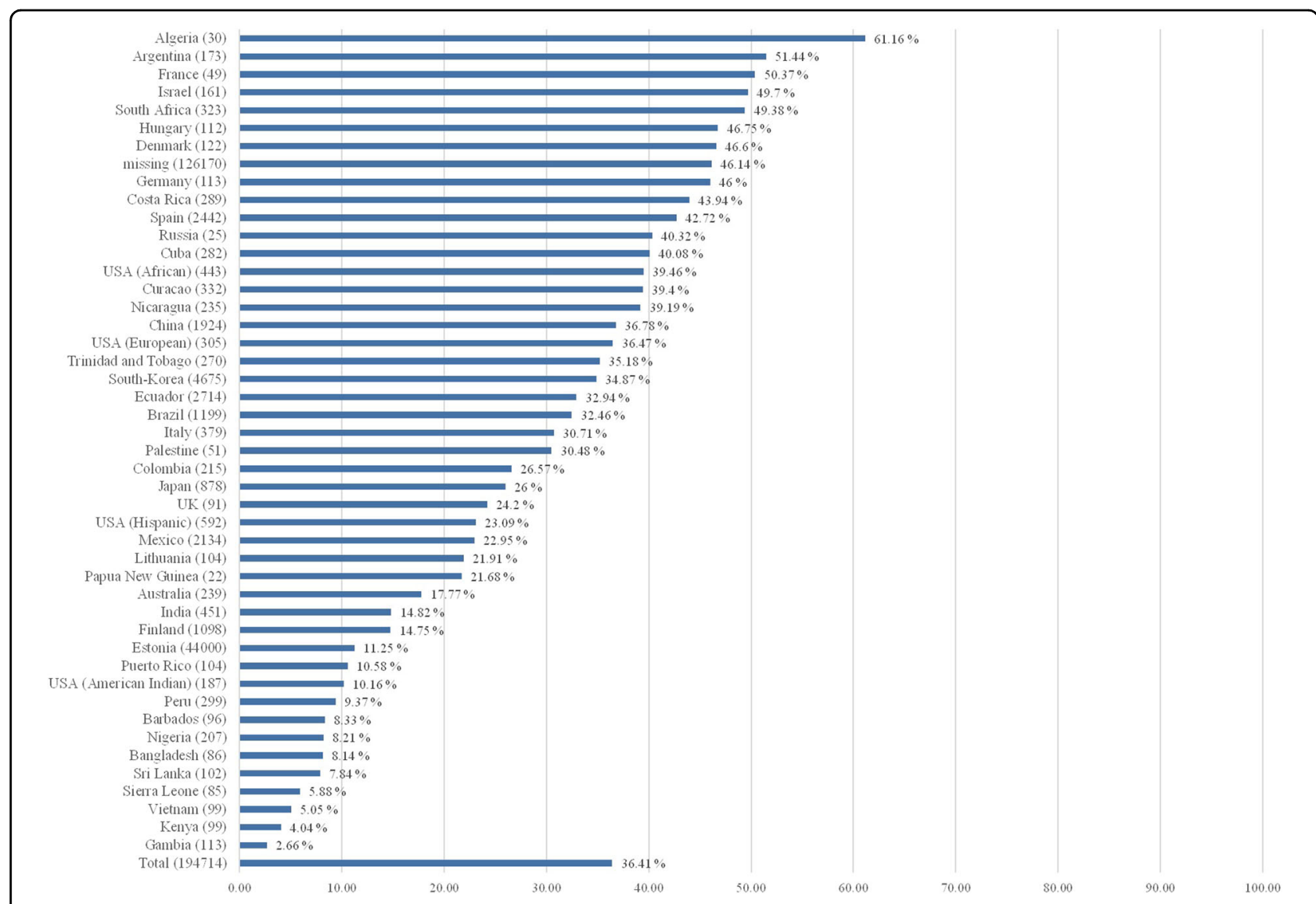

Fig. 2 CYP2D6 non-normal probability estimate per country. Country (genotyped subjects); non-normal probability estimate in percentage.

probability estimate $69.2 \%)^{64,65}$. The probability is lowest in countries in the Americas, particularly Mexico (nonNM probability estimate $31.7 \%)^{66-68}$ and Costa Rica (nonNM probability estimate $33.9 \%)^{69}$. CYP2C19 non-NM probability estimates are shown in Fig. 4, and Fig. 5 displays CYP2C19 non-NM probability estimates on a world map.

\section{Discussion}

In this meta-analysis, we introduce a clinical useful concept of a non-NM probability estimate as the equivalent of the sum-prevalence (in percentages) of PM + IM + UM predicted CYP2D6 and CYP2C19 phenotypes. The mean totals of non-NM probability estimates worldwide were $36.4 \%$ (CYP2D6) and 61.9\% (CYP2C19). This means that more than half of all psychiatric patients have a nonnormal predicted CYP2D6 and/or CYP2C19 metabolizer phenotype. Since $>75 \%$ of the psychopharmacological drugs are metabolized by one or both of these enzymes ${ }^{6,70}$, the results of our meta-analysis emphasize the importance of integrating pharmacogenetic information into clinical practice, especially when treating patients who have had adverse drug events or show treatment resistance.
We included a total of 318 studies in our meta-analysis of CYP2D6 and CYP2C19 genotypes in healthy populations of which genotype requirements were fulfilled by 118 studies (37\%). This is an important requirement, because having too few CYP2D6 or CYP2C19 alleles in a study may not accurately determine predicted phenotype from genotype data.

\section{Diversity within major regions}

Although grouping populations can simplify reporting of pharmacogenetic alleles, we grouped ethnicities within geographical regions to enable comparisons with other published meta-analyses ${ }^{71,72}$. The prevalences of our CYP2D6 predicted phenotypes were in general agreement with those reported by Gaedigk et al. ${ }^{17}$. However, our data predicted a higher percentage of UMs for Africans (i.e., $5.5 \%, n=562$ versus $3.8 \%, n=430$ ). This difference may be explained by the fact that we weighted the number of genotyped subjects when calculating the mean allele frequencies for our meta-analysis. In Gaedigk et al.'s report, studies were not weighted by sample size, so small studies might have had a bigger influence on the mean. We also included two studies in Africans that were not included by 


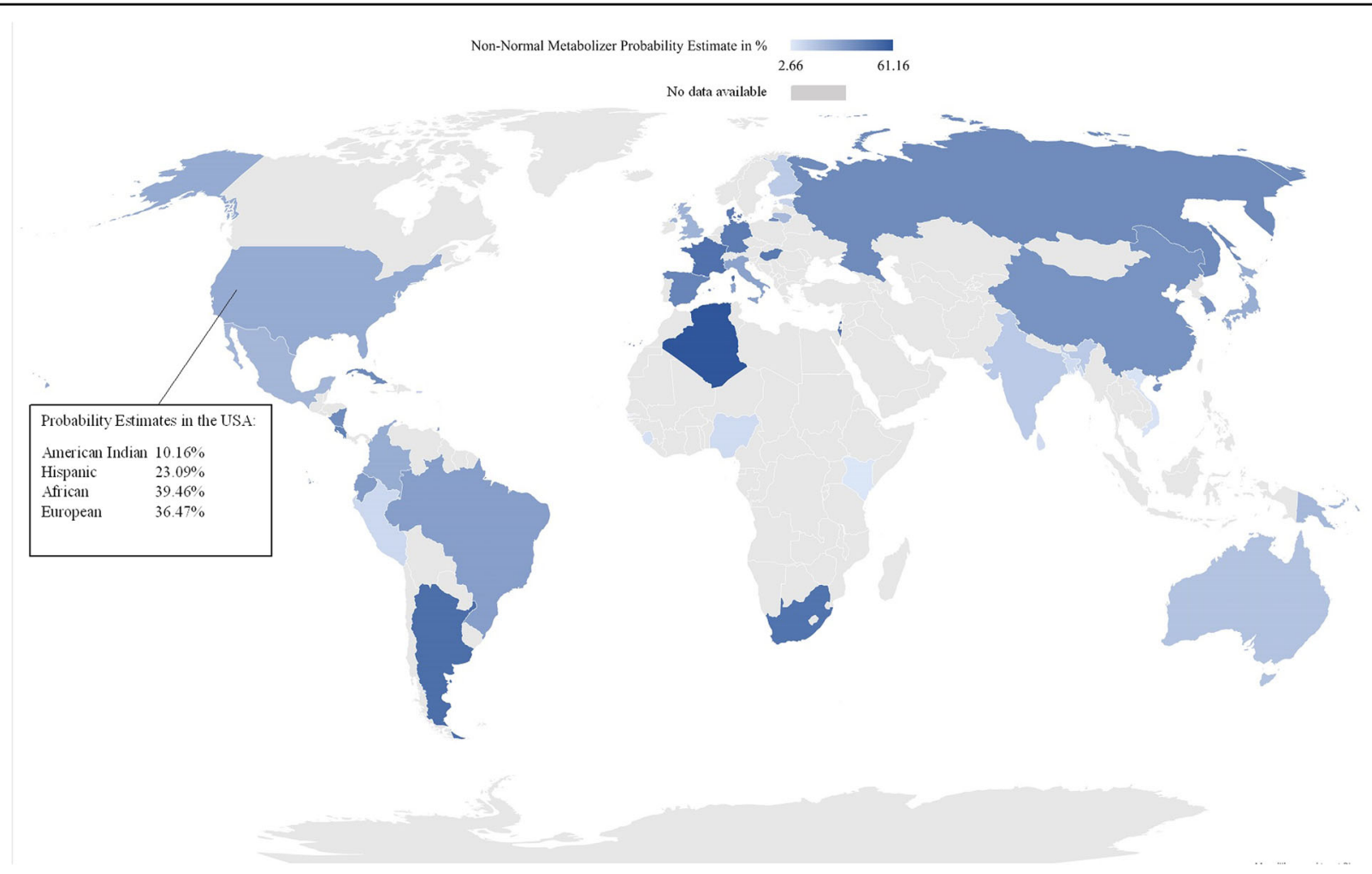

Fig. 3 Worldwide CYP2D6 non-normal metabolizer probability estimates.

Gaedigk et al. due to their small sample size and thus not meeting their requirements of at least 50 study subjects; they both reported higher frequencies of $\mathrm{UMs}^{48,73}$. In contrast, in the South Central Asian population the percentage of UMs found by Gaedigk et al. was higher than those reported here, $2.8 \%(n=370)$ vs. $2.1 \%(n=434)$, due to the inclusion of two studies in South East Asians in this meta-analysis, which found no $\mathrm{UMs}^{73,74}$.

In contrast with Fricke-Galindo et al. ${ }^{19}$ and the CPIC's CYP2C19 allele frequency table (https://www.pharmgkb. org/page/cyp2c19RefMaterials), we found a much higher prevalence of $25.0 \%$ for CYP2C19 UMs in Oceania (vs. 0\% and $1.5 \%)$. This is because we only included studies investigating the CYP2C19*17 increased function allele, which leads to rapid and ultrarapid metabolizer predicted phenotypes. Studies that do not investigate this allele yield underestimates of the prevalence of UMs.

\section{Clinical practice}

Some issues need to be addressed in translating genotype data to information useful for clinical practice. Although prevalences of non-NM are high, there is no conclusive evidence whether $C Y P$ genotyping is beneficial for clinical outcomes in psychiatric practice. There are a few prospective studies analyzing the clinical utility of $C Y P$ genotyping and they report contradictory outcomes in diverse populations ${ }^{27-29}$. So far, it is still unclear which patient groups might benefit from genotyping and see better treatment outcomes. One reason for the uncertainty is the possibility of the transformation of genotypic EMs into phenotypic PMs by multiple causes, for example, due to smoking, CYP2D6 and CYP2C19 inhibiting medication (e.g., bupropion and esomeprazole), CYP2D6 and CYP2C19 inducing medication (e.g., oritavancin and carbamazepine), and inflammation or co-morbidities ${ }^{75,76}$. This phenomenon is called phenoconversion: it has been described in diverse populations ${ }^{77-79}$. If this is happening on a large scale, it means genotypic outcomes could be unreliable for use in clinical practice. It may already influence outcomes of studies on the effectivity of $C Y P$ genotyping in clinical practice ${ }^{28}$. Another issue may arise with genotyping patients who have been on treatment for several years, because their brain has adapted to the changed levels of neurotransmitters and the side effects are no longer reversible ${ }^{80,81}$.

Lastly, the category of the IM has been the subject of debate $^{82}$. In this meta-analysis, we categorized the IM as defined by the $\mathrm{CPIC}^{47}$. Because IMs only show minor differences in metabolism from $\mathrm{NMs}^{83}$, one could categorize them in the NM group. However, other studies have indicated that IMs show lower oral drug clearance, higher blood serum levels, and have higher chances of side 
Table 4 Mean frequencies of CYP2C19 predicted phenotypes per ethnicity ${ }^{\mathrm{a}}$.

\begin{tabular}{|c|c|c|c|c|c|c|c|c|c|c|}
\hline \multirow[t]{2}{*}{ Ethnicity } & \multirow[b]{2}{*}{$\mathbf{n}$} & \multicolumn{2}{|c|}{ PM } & \multicolumn{2}{|c|}{ IM } & \multicolumn{2}{|c|}{ NM } & \multicolumn{2}{|c|}{ UM } & \multirow{2}{*}{$\frac{\text { Others }}{\mathbf{M}}$} \\
\hline & & $\mathbf{M}$ & SD & $\mathbf{M}$ & SD & $\mathbf{M}$ & SD & M & SD & \\
\hline \multicolumn{11}{|l|}{ Africa } \\
\hline African & 5203 & 0.033 & 0.000 & 0.310 & 0.000 & 0.344 & 0.000 & 0.335 & 0.000 & 0.000 \\
\hline $\begin{array}{l}\text { Cape Mixed Ancestry } \\
\text { (South African) }\end{array}$ & 75 & 0.080 & 0.000 & 0.400 & 0.000 & 0.350 & 0.000 & 0.170 & 0.000 & 0.000 \\
\hline Tzotziles (Ugandese) & 99 & I 0.020 & 0.000 & 0.230 & 0.000 & 0.510 & 0.000 & 0.240 & 0.000 & 0.000 \\
\hline Xhosa (South African) & 100 & 0.030 & 0.000 & 0.490 & 0.000 & 0.390 & 0.000 & 0.090 & 0.000 & 0.000 \\
\hline African American & 3533 & 0.039 & 0.007 & 0.308 & 0.001 & 0.337 & 0.013 & 0.316 & 0.020 & 0.001 \\
\hline \multicolumn{11}{|l|}{ Americas } \\
\hline $\begin{array}{l}\text { Admixed Latin Americans } \\
\text { African Caribbeans from }\end{array}$ & 5789 & | 0.011 & 0.000 & 0.192 & 0.000 & 0.661 & 0.000 & 0.210 & 0.000 & 0.000 \\
\hline Barbados (Costa Rica) & 46 & 0.000 & 0.000 & 0.315 & 0.000 & 0.345 & 0.000 & 0.326 & 0.000 & 0.014 \\
\hline Brazilian & 1043 & | 0.018 & 0.000 & 0.230 & 0.000 & 0.482 & 0.000 & 0.270 & 0.000 & 0.000 \\
\hline Bribri (Costa Rican) & 23 & 0.000 & 0.000 & 0.082 & 0.000 & 0.834 & 0.000 & 0.087 & 0.000 & 0.000 \\
\hline Costa Rican & 36 & 0.000 & 0.000 & 0.219 & 0.000 & 0.717 & 0.000 & 0.056 & 0.000 & 0.008 \\
\hline Dutch Caribbean & 332 & 0.041 & 0.000 & 0.241 & 0.000 & 0.372 & 0.000 & 0.345 & 0.000 & 0.001 \\
\hline Ecuadorian & 139 & 0.008 & 0.000 & 0.243 & 0.000 & 0.591 & 0.000 & 0.159 & 0.000 & 0.000 \\
\hline Guarani (Brazil) & 90 & | 0.012 & 0.000 & 0.197 & 0.000 & 0.771 & 0.000 & | 0.019 & 0.000 & 0.000 \\
\hline Guaymi (Costa Rican) & 24 & 0.000 & 0.000 & 0.000 & 0.000 & 0.960 & 0.000 & I 0.040 & 0.000 & 0.000 \\
\hline Mestizo (Costa Rican) & 141 & 0.000 & 0.000 & 0.144 & 0.000 & 0.671 & 0.000 & 0.177 & 0.000 & 0.008 \\
\hline Mestizo (Ecuadorian) & 297 & | 0.007 & 0.000 & 0.151 & 0.000 & 0.428 & 0.000 & 0.414 & 0.000 & 0.000 \\
\hline Mestizo (Mexican) & 300 & 0.000 & 0.000 & 0.170 & 0.000 & 0.660 & 0.000 & 0.170 & 0.000 & 0.000 \\
\hline Mexican & 568 & | 0.014 & 0.007 & 0.208 & 0.007 & 0.696 & 0.070 & 0.082 & 0.084 & 0.000 \\
\hline \multicolumn{11}{|l|}{ Central/ South East Asia } \\
\hline Buruhi (Pakistani) & 118 & 0.060 & 0.000 & 0.600 & 0.000 & 0.200 & 0.000 & 0.140 & 0.000 & 0.000 \\
\hline Burushu (Pakistani) & 28 & 0.000 & 0.000 & 0.150 & 0.000 & 0.460 & 0.000 & 0.390 & 0.000 & 0.000 \\
\hline Hazara (Pakistani) & 102 & 0.000 & 0.000 & 0.410 & 0.000 & 0.270 & 0.000 & 0.320 & 0.000 & 0.000 \\
\hline Indian (West) & 102 & 0.123 & 0.000 & 0.455 & 0.000 & 0.299 & 0.000 & 0.123 & 0.000 & 0.000 \\
\hline Kalash (Pakistani) & 64 & 0.050 & 0.000 & 0.560 & 0.000 & 0.280 & 0.000 & 0.110 & 0.000 & 0.000 \\
\hline Koya (Indian) & 460 & 0.152 & 0.000 & 0.499 & 0.000 & 0.150 & 0.000 & 0.197 & 0.000 & 0.003 \\
\hline Naik (Indian) & 100 & 0.310 & 0.000 & 0.450 & 0.000 & 0.130 & 0.000 & 0.110 & 0.000 & 0.000 \\
\hline Pakistani & 685 & 0.121 & 0.013 & 0.452 & 0.014 & 0.184 & 0.047 & 0.243 & 0.020 & 0.000 \\
\hline Parsi (Pakistani) & 90 & 0.000 & 0.000 & 0.320 & 0.000 & 0.300 & 0.000 & 0.380 & 0.000 & 0.000 \\
\hline Pathan (Pakistani) & 170 & 0.070 & 0.000 & 0.460 & 0.000 & 0.320 & 0.000 & 0.150 & 0.000 & 0.000 \\
\hline Punjabi (Pakistani) & 218 & 0.070 & 0.000 & 0.460 & 0.000 & 0.280 & 0.000 & 0.190 & 0.000 & 0.000 \\
\hline Saraiki (Pakistani) & 59 & 0.200 & 0.000 & 0.400 & 0.000 & 0.300 & 0.000 & 0.100 & 0.000 & 0.000 \\
\hline Sindhi (Pakistani) & 179 & 0.089 & 0.000 & 0.453 & 0.000 & 0.352 & 0.000 & 0.106 & 0.000 & 0.000 \\
\hline South Asian & 8256 & 0.118 & 0.000 & 0.458 & 0.000 & 0.280 & 0.000 & 0.162 & 0.000 & 0.000 \\
\hline Tamil (Indian) (South) & 206 & 0.184 & 0.000 & 0.310 & 0.000 & 0.288 & 0.000 & 0.219 & 0.000 & 0.000 \\
\hline \multicolumn{11}{|l|}{ East Asia } \\
\hline Bai (Chinese) & 202 & 0.095 & 0.000 & 0.425 & 0.000 & 0.473 & 0.000 & 0.000 & 0.000 & 0.006 \\
\hline Chinese & 754 & 0.127 & 0.025 & 0.463 & 0.008 & 0.410 & 0.016 & 0.001 & 0.003 & 0.000 \\
\hline East Asian & 5407 & 0.142 & 0.013 & 0.463 & 0.019 & 0.380 & 0.013 & | 0.021 & 0.011 & 0.000 \\
\hline Hakka (Chinese) & 6686 & 0.131 & 0.000 & 0.452 & 0.000 & 0.417 & 0.000 & 0.000 & 0.000 & 0.000 \\
\hline Han (Chinese) & 1972 & 0.144 & 0.043 & 0.437 & 0.038 & 0.416 & 0.064 & 0.002 & 0.009 & 0.002 \\
\hline Han (Chinese) (North) & 1000 & 0.137 & 0.000 & 0.488 & 0.000 & 0.373 & 0.000 & 0.000 & 0.000 & 0.002 \\
\hline Han (Chinese) (South) & 1127 & 0.146 & 0.000 & 0.502 & 0.000 & 0.349 & 0.000 & 0.000 & 0.000 & 0.003 \\
\hline Han (Taiwanese) & 180 & 0.118 & 0.000 & 0.516 & 0.000 & 0.365 & 0.000 & 0.000 & 0.000 & 0.001 \\
\hline Hui (Chinese) & 265 & 0.280 & 0.023 & 0.441 & 0.070 & 0.279 & 0.093 & 0.000 & 0.000 & 0.000 \\
\hline Japanese & 2577 & 0.175 & 0.038 & 0.488 & 0.038 & 0.333 & 0.040 & 0.003 & 0.005 & 0.001 \\
\hline Kazakh (Chinese) & 107 & 0.075 & 0.000 & 0.318 & 0.000 & 0.607 & 0.000 & 0.000 & 0.000 & 0.000 \\
\hline Li (Chinese) & 265 & 0.117 & 0.039 & 0.439 & 0.043 & 0.443 & 0.081 & 0.000 & 0.000 & 0.000 \\
\hline Mongolian & 553 & 0.123 & 0.056 & 0.427 & 0.047 & 0.450 & 0.099 & 0.000 & 0.000 & 0.000 \\
\hline Shanghai (Chinese) & 96 & 0.135 & 0.000 & 0.385 & 0.000 & 0.469 & 0.000 & 0.000 & 0.000 & 0.011 \\
\hline Shantou (Chinese) & 96 & 0.073 & 0.000 & 0.365 & 0.000 & 0.510 & 0.000 & | 0.021 & 0.000 & 0.031 \\
\hline Shenyang (Chinese) & 96 & 0.042 & 0.000 & 0.327 & 0.000 & 0.598 & 0.000 & 0.033 & 0.000 & 0.000 \\
\hline South Korean & 1962 & 0.131 & 0.020 & 0.474 & 0.030 & 0.391 & 0.030 & 0.003 & 0.007 & 0.000 \\
\hline Tibetan Chinese & 96 & 0.041 & 0.000 & 0.406 & 0.000 & 0.563 & 0.000 & | 0.031 & 0.000 & 0.000 \\
\hline Tohoku (Japanese) & 57 & 0.189 & 0.000 & 0.575 & 0.000 & 0.213 & 0.000 & 0.000 & 0.000 & 0.023 \\
\hline Uyghurs (Chinese) & 459 & 0.095 & 0.035 & 0.382 & 0.070 & 0.530 & 0.105 & 0.000 & 0.000 & 0.000 \\
\hline Xi'an (Chinese) & 96 & 0.146 & 0.000 & 0.313 & 0.000 & 0.521 & 0.000 & | 0.010 & 0.000 & 0.010 \\
\hline
\end{tabular}


Table 4 continued

\begin{tabular}{|c|c|c|c|c|c|c|c|c|c|c|}
\hline \multicolumn{11}{|l|}{ Europe } \\
\hline Avars (Russian) & 90 & | 0.016 & 0.000 & 0.222 & 0.000 & 0.453 & 0.000 & 0.309 & 0.000 & 0.000 \\
\hline Danish & 276 & | 0.022 & 0.000 & 0.258 & 0.000 & 0.442 & 0.000 & 0.279 & 0.000 & 0.000 \\
\hline Dargins (Russian) & 50 & 0.003 & 0.000 & 0.095 & 0.000 & 0.504 & 0.000 & 0.398 & 0.000 & 0.000 \\
\hline Estonian & 44000 & | 0.024 & 0.000 & 0.236 & 0.000 & 0.359 & 0.000 & 0.381 & 0.000 & 0.000 \\
\hline European & 96882 & | 0.027 & 0.005 & 0.274 & 0.020 & 0.374 & 0.012 & 0.329 & 0.008 & 0.000 \\
\hline European (USA) & 17810 & | 0.023 & 0.002 & 0.255 & 0.005 & 0.410 & 0.010 & 0.311 & 0.007 & 0.000 \\
\hline Faroese & 311 & 0.032 & 0.000 & 0.309 & 0.000 & 0.460 & 0.000 & 0.199 & 0.000 & 0.000 \\
\hline Finnish (North) & 497 & $\begin{array}{l}0.003 \\
\end{array}$ & 0.000 & 0.111 & 0.000 & 0.585 & 0.000 & 0.300 & 0.000 & 0.000 \\
\hline Greek & 283 & | 0.021 & 0.000 & 0.219 & 0.000 & 0.442 & 0.000 & 0.318 & 0.000 & 0.000 \\
\hline Hispanic (USA) & 3509 & | 0.021 & 0.000 & 0.226 & 0.006 & 0.531 & 0.007 & 0.223 & 0.001 & 0.000 \\
\hline Jewish & 250 & 0.044 & 0.000 & 0.252 & 0.000 & 0.416 & 0.000 & 0.288 & 0.000 & 0.000 \\
\hline Jewish (Ashkenazi) & 5326 & | 0.023 & 0.001 & 0.253 & 0.010 & 0.457 & 0.013 & 0.264 & 0.005 & 0.004 \\
\hline Jewish (Sephardi) & 135 & | 0.012 & 0.000 & 0.160 & 0.000 & 0.522 & 0.000 & 0.267 & 0.000 & 0.039 \\
\hline Laks (Russian) & 46 & 0.034 & 0.000 & 0.300 & 0.000 & 0.372 & 0.000 & 0.294 & 0.000 & 0.000 \\
\hline Macedonian & 184 & | 0.027 & 0.000 & 0.234 & 0.000 & 0.418 & 0.000 & 0.321 & 0.000 & 0.000 \\
\hline Nanai (Russian) & 70 & 0.108 & 0.000 & 0.442 & 0.000 & 0.423 & 0.000 & | 0.028 & 0.000 & 0.000 \\
\hline Norwegian & 309 & | 0.013 & 0.000 & 0.279 & 0.000 & 0.395 & 0.000 & 0.314 & 0.000 & 0.000 \\
\hline Scandinavian & 394 & | 0.020 & 0.000 & 0.242 & 0.000 & 0.450 & 0.000 & 0.288 & 0.000 & 0.000 \\
\hline Spanish (North) & 282 & | 0.018 & 0.000 & 0.213 & 0.000 & 0.500 & 0.000 & 0.269 & 0.000 & 0.000 \\
\hline Swedish & 185 & || 0.026 & 0.000 & 0.269 & 0.000 & 0.410 & 0.000 & 0.296 & 0.000 & 0.000 \\
\hline \multicolumn{11}{|l|}{ Middle East } \\
\hline Caspian (Iranian) & 73 & | 0.012 & 0.000 & 0.194 & 0.000 & 0.518 & 0.000 & 0.275 & 0.000 & 0.000 \\
\hline Fars (Iranian) & 180 & 0.033 & 0.000 & 0.298 & 0.000 & 0.287 & 0.000 & 0.382 & 0.000 & 0.000 \\
\hline Iranian & 232 & 0.030 & 0.015 & 0.224 & 0.014 & 0.414 & 0.005 & 0.331 & 0.023 & 0.002 \\
\hline Kurd (Iranian) & 95 & 0.077 & 0.000 & 0.401 & 0.000 & 0.259 & 0.000 & 0.264 & 0.000 & 0.000 \\
\hline Lure (Iranian) & 80 & 0.127 & 0.000 & 0.459 & 0.000 & 0.033 & 0.000 & 0.381 & 0.000 & 0.000 \\
\hline Saudi Arabian & 393 & 0.005 & 0.001 & 0.150 & 0.001 & 0.603 & 0.178 & 0.242 & 0.176 & 0.001 \\
\hline Turk (Iranian) & 110 & 0.072 & 0.000 & 0.392 & 0.000 & 0.214 & 0.000 & 0.321 & 0.000 & 0.000 \\
\hline Turkish & 369 & 0.027 & 0.031 & 0.163 & 0.186 & 0.485 & 0.206 & 0.325 & 0.011 & 0.000 \\
\hline \multicolumn{11}{|l|}{ Oceania } \\
\hline Pacific Islander & 24 & 0.000 & 0.000 & 0.167 & 0.000 & 0.583 & 0.000 & 0.250 & 0.000 & 0.000 \\
\hline Total & 225173 & 0.042 & 0.041 & 0.290 & 0.079 & 0.386 & 0.071 & 0.287 & 0.111 & 0.000 \\
\hline
\end{tabular}

$M$ mean, $n$ number of genotyped subjects, $S D$ standard deviation, $P M$ poor metabolizers, $I M$ intermediate metabolizers, $N M$ normal metabolizers, UM ultrarapid metabolizers.

${ }^{\mathrm{a}}$ For references, please see Supplemental Table 3.

${ }^{\mathrm{b}}$ An SNP combination that could not be assigned to a known allele/phenotype.

effects than $\mathrm{NMs}^{84-86}$. For this reason, we consider that the IM status is clinically relevant for psychiatric patients and guidelines for some medications are now advising dose adjustments for this predicted phenotype $\mathrm{e}^{25,87}$. Estimations of the worldwide prevalence of only $\mathrm{PM}+\mathrm{UM}$ were $7.75 \%$ (CYP2D6) and 32.94\% (CYP2C19) (Supplemental Figs. 1 and 2).

\section{Strengths and limitations}

We included studies with a small number of participants $(n \geq 20)$, as well as controls from case-control studies, which increased the number of studies we could include. Bias was minimized by excluding studies of populations diagnosed with a disease to prevent confounding our data (i.e., disease associations with specific allele frequencies ${ }^{88-94}$ ). On the other hand, some large studies had to be excluded due to their inclusion of non- healthy individuals, which might have biased the outcomes of some countries ${ }^{95}$.

Another strength is that we used region-specific inclusion criteria to maximize the accuracy of the phenotype predictions ${ }^{17,19,26}$. This helped to avoid applying criteria based on studies in Western countries to other regions of the world. This led to the exclusion of studies reporting on too few allelic variants and of studies focusing on the determination of only PM or UM in a population $^{18,96,97}$.

Studies reporting allele frequencies of merely CYP2C19*2 and *3 or studies investigating CYP2D6 allele duplications, but with no minimum set of variants, are certainly of scientific importance but not of practical importance for clinicians because no complete risk inventory of the metabolizer phenotype could be determined. Because we excluded studies not investigating 


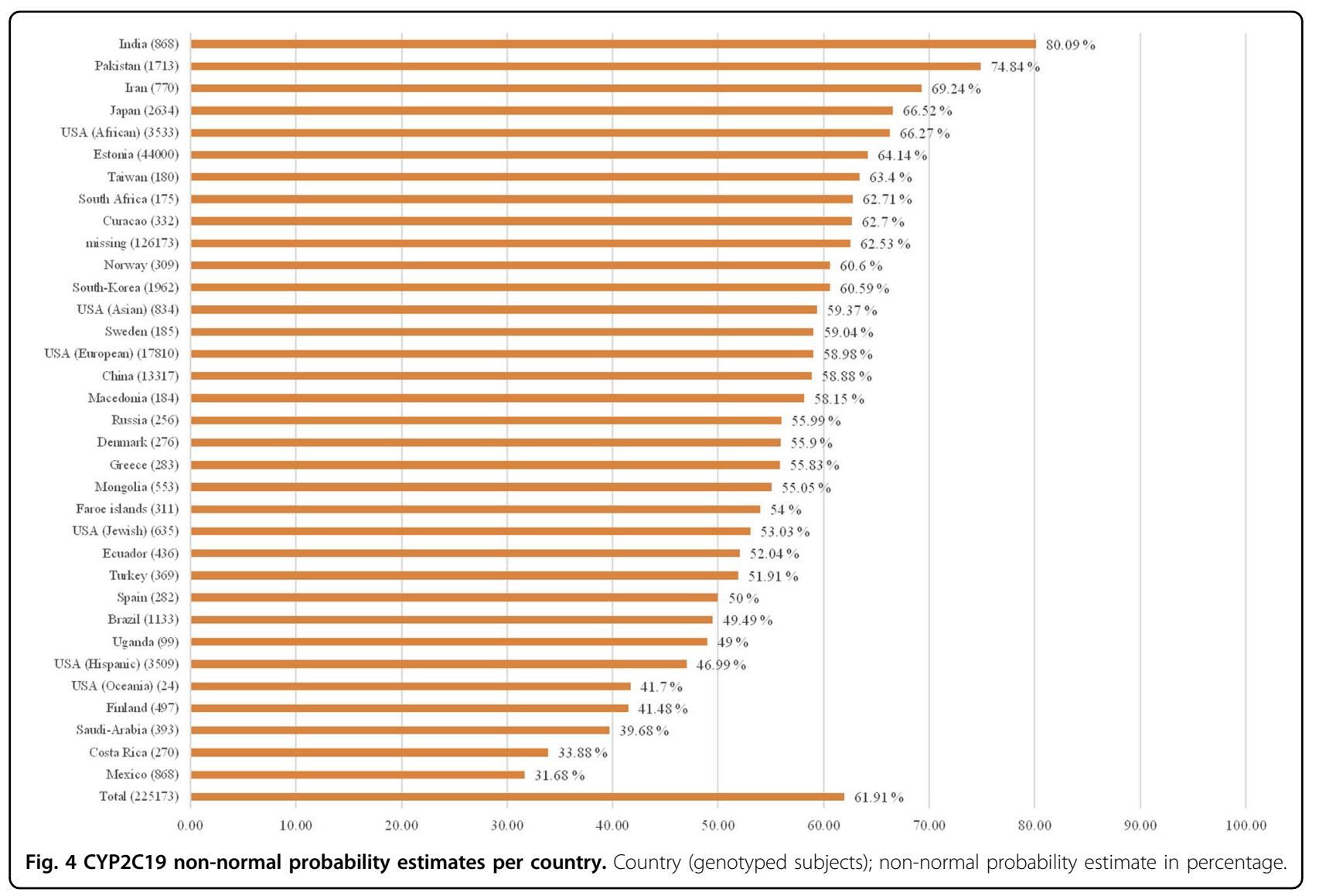

CYP2C19*17, we had only one report describing Oceanians $(n=24)^{98}$, which did not identify any PMs.

A limitation is that we were depended on the sensitivity of the tests of the individual studies. For example, because of overlap in single-nucleotide polymorphisms in the CYP2D6*10 and *36 and in the CYP2D6*17 and *40 allele, a slight overestimation or underestimation of some predicted phenotypes might have been reported in some studies ${ }^{99}$.

The inclusion of studies with a small number of participants (20-50) could have led to an overestimation or underestimation of predicted phenotypes in some populations, but the influence on the mean prevalence was minimized by weighting the number of genotyped subjects. We may still have made overestimates or underestimates where there are few studies for a certain region/country along with a relatively small number of studied subjects. Because few studies reporting specific minority ethnicities met our inclusion criteria, we did not want to exclude potentially valuable information from our meta-analysis by setting too-stringent participant number requirements.

Although we only included studies on homogenous ethnic groups in this meta-analysis, we are aware of the limitations of grouping ethnicities based on self-reported ethnicity. Although ancestry based on genetic information is more objective than self-reported ethnicity, much of the research into CYP genotypes has been based on selfreported ethnicity, while for a few minority populations, some genetic data were systematically analyzed. In a study of 103,006 participants with 23 ethnicities, a very high correspondence was found between self-reported ethnicity and genetic ancestry ${ }^{100}$. Only African Americans and Latino Americans demonstrated a higher degree of ancestral admixture than self-reported.

Second, although studies of genetic ancestry show there is a strong linkage between belonging to an ethnic group and coming from a certain geographical region, ethnicity is not always the same as geographical region ${ }^{44}$. Ethnic groups migrate, and although some ethnicities show almost no admixture with the local ethnicity even many years after migration, other ethnicities do show a mixture of multiple ancestors.

Especially in countries in the Americas, North America, and Canada, ethnic backgrounds can be diverse and individual ethnicity is increasingly blurred by admixture, making self-reported ethnic background or geographical location less predictive for a correct estimation on a nonNM predicted phenotype ${ }^{76}$. The probability estimates per country (Figs. 2-5) are means of the probability estimates 


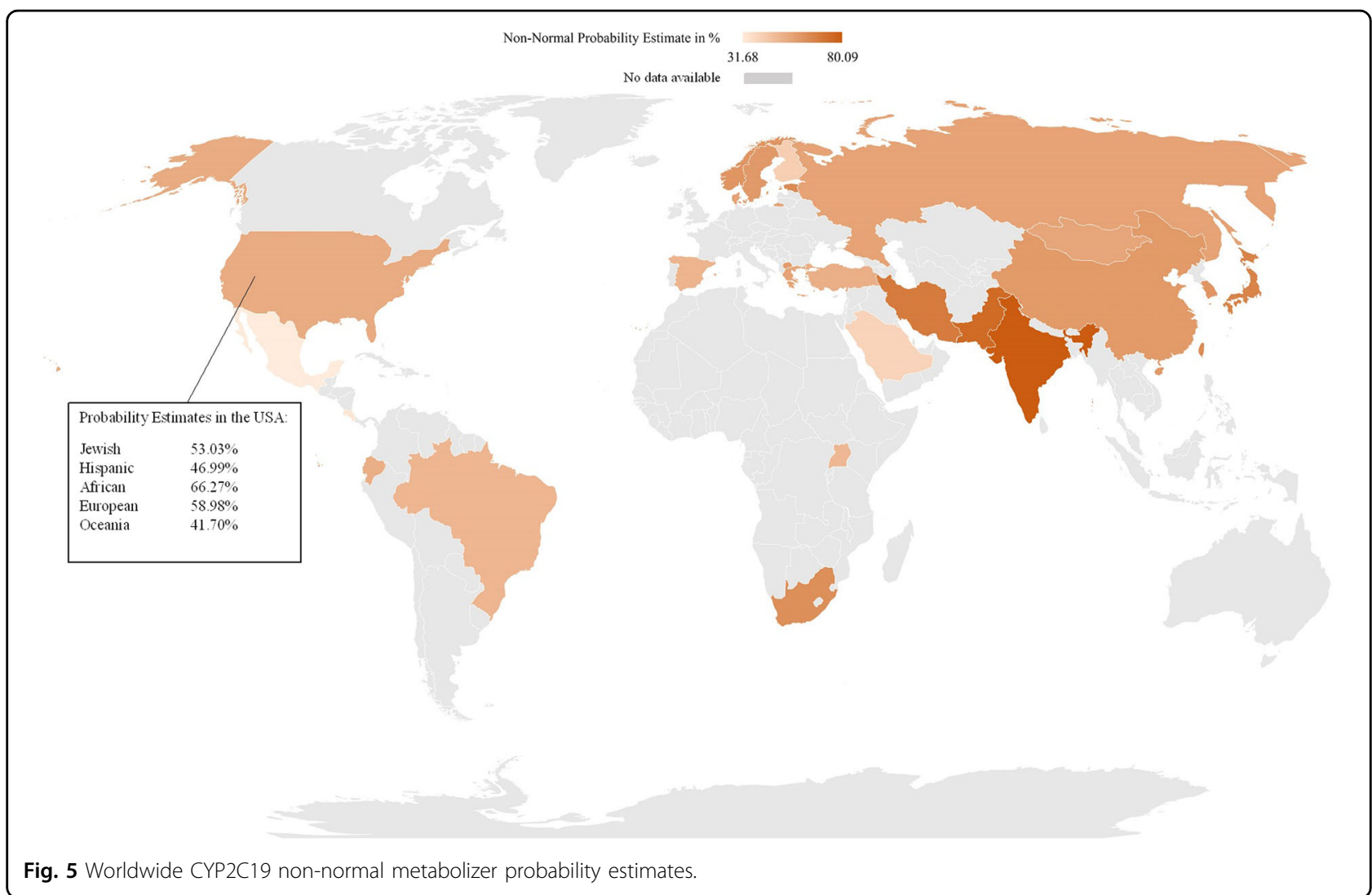

of these different ethnicities and must therefore be interpreted with caution.

The total means are mean probability estimates of all the included populations and represent a worldwide mean probability estimate. Because countries were not weighted by the number of inhabitants, small countries with large study populations have a relatively large influence on the estimated mean. In addition, some geographical regions were significantly underinvestigated (Africa and the Middle East) and their predicted phenotype distributions are not adequately represented in the total estimated means.

\section{Conclusions}

In this comprehensive meta-analysis of worldwide CYP2D6 and CYP2C19 genotype variation, (>336,000 subjects, 318 reports), we found that the mean total probability estimates for a non-NM predicted phenotype are $36.4 \%$ for CYP2D6 and $61.9 \%$ for CYP2C19. The estimates reveal a large geographical variation (3-61\% and $32-80 \%$, respectively). Our results suggest that more than half of the world population has a nonnormal CYP2D6 and/or CYP2C19 metabolizer predicted phenotype. Based on the documented probability estimates, pre-emptive pharmacogenetic testing is encouraged for every patient who will undergo therapy with a drug(s) that is metabolized by CYP2D6 and/or CYP2C19 pathways and should be considered in case of treatment resistance or serious side effects.

Second, many of the studies were not relevant for clinical practice, because they only investigated a minimum number of allelic variants and thus any phenotype prediction is unlikely to be accurate. Especially when estimating the prevalence of the CYP2C19 UM predicted phenotype, studies in all regions except for East Asia should genotype on "17 to come to a reliable phenotype prediction. We therefore recommend that, when allele frequencies are being studied, a minimum number of alleles-depending on the geographical region-must be assessed to be able to predict phenotypes as accurately as possible $^{101}$.

\section{Acknowledgements}

We thank Andrea Gaedigk for critical discussion regarding the content. We thank Tale Evenhuis of the University of Leiden and all the members of the library of Parnassia Psychiatric Institute for assisting in the search and collection of articles. In addition, we thank Petra Burgisser for helping import the data into our database and Mathijs Deen for assisting with the statistics. We thank Judith Offringa for editing a near-final version of the manuscript.

\section{Author details}

${ }^{1}$ Parnassia Academy, Parnassia Psychiatric Institute, The Hague, the Netherlands. ${ }^{2}$ School for Mental Health and Neuroscience, Maastricht University, Maastricht, the Netherlands. ${ }^{3}$ Department of Psychiatric Residency Training, Pro Persona Mental Health, Wolfheze, the Netherlands. ${ }^{4}$ Department 
of Criminal Law, Tilburg Law School, Tilburg University, Tilburg, the Netherlands. ${ }^{5}$ Department of Psychiatry, University Medical Center Groningen, University of Groningen, Groningen, the Netherlands. ${ }^{6}$ Mailman School of Public Health, Columbia University, New York, NY, USA. ' Innova, Psychiatric Centre GGZ Centraal, Amersfoort, The Netherlands

\section{Author contributions}

Study conception and design: all authors. Acquisition of data: A.B.K., D.J.V. Analysis and interpretation of data: A.B.K., D.J.V., M.B. Drafting of manuscript: A.B.K. Critical revision: D.V., M.B., P.N.v.H., H.W.H.

\section{Conflict of interest}

The authors declare that they have no conflict of interest.

\section{Publisher's note}

Springer Nature remains neutral with regard to jurisdictional claims in published maps and institutional affiliations.

Supplementary information accompanies this paper at (https://doi.org/ 10.1038/s41398-020-01129-1).

Received: 5 May 2020 Revised: 17 November 2020 Accepted: 1 December 2020

Published online: 24 February 2021

\section{References}

1. Bhugra, D. et al. WPA guidance on mental health and mental health care in migrants. World Psychiatry 10, 2-10 (2011).

2. Perlis, R. H. Abandoning personalization to get to precision in the pharmacotherapy of depression. World Psychiatry 15, 228-235 (2016).

3. Kirchheiner, J. et al. Pharmacogenetics of antidepressants and antipsychotics: the contribution of allelic variations to the phenotype of drug response. Mol. Psychiatry 9, 442-473 (2004).

4. Fleeman, N. et al. Cytochrome P450 testing for prescribing antipsychotics in adults with schizophrenia: systematic review and meta-analyses. Pharmacogenomics J. 11, 1-14 (2011).

5. Ingelman-Sundberg, M. Pharmacogenetics of cytochrome P450 and its applications in drug therapy: the past, present and future. Trends Pharmacol. Sci. 25, 193-200 (2004).

6. Weide, J. \& van der, Hinrichs, J. W. J. The influence of cytochrome P450 pharmacogenetics on disposition of common antidepressant and antipsychotic medications. Clin. Biochem. Rev. 27, 17-25 (2006).

7. Arranz, M. J. \& de Leon, J. Pharmacogenetics and pharmacogenomics of schizophrenia: a review of last decade of research. Mol. Psychiatry 12, 707-747 (2007).

8. van der Weide, J. \& Steijns, L. S. Cytochrome P450 enzyme system: genetic polymorphisms and impact on clinical pharmacology. Ann. Clin. Biochem. 36 722-729 (1999).

9. Kishimoto, T., Hagi, K., Nitta, M., Kane, J. M. \& Correll, C. U. Long-term effectiveness of oral second-generation antipsychotics in patients with schizophrenia and related disorders: a systematic review and meta-analysis of direct head-to-head comparisons. World Psychiatry 18, 208-224 (2019).

10. de Leon, J. et al. The CYP2D6 poor metabolizer phenotype may be associated with risperidone adverse drug reactions and discontinuation. J. Clin. Psychiatry 66, 15-27 (2005).

11. Patsopoulos, N. A., Ntzani, E. E., Zintzaras, E. \& loannidis, J. P. A. CYP2D6 polymorphisms and the risk of tardive dyskinesia in schizophrenia: a metaanalysis. Pharmacogenet. Genomics 15, 151-158 (2005).

12. Brockmoller, J. et al. The impact of the polymorphism on haloperidol pharmacokinetics and on the outcome of haloperidol treatment. Clin. Pharmacol. Ther. 72, 438-452 (2002).

13. Lisbeth, P. et al. Genotype and co-medication dependent CYP2D6 metabolic activity: effects on serum concentrations of aripiprazole, haloperidol, risperidone, paliperidone and zuclopenthixol. Eur. J. Clin. Pharmacol. 72, 175-184 (2016).

14. Schenk, P. W. et al. The CYP2C19*17 genotype is associated with lower imipramine plasma concentrations in a large group of depressed patients. Pharmacogenomics J. 10, 219-225 (2010).
15. Kawanishi, C., Lundgren, S., Ågren, H. \& Bertilsson, L. Increased incidence of CYP2D6 gene duplication in patients with persistent mood disorders: ultrarapid metabolism of antidepressants as a cause of nonresponse. A pilot study. Eur. J. Clin. Pharmacol. 59, 803-807 (2004).

16. Bradford, L. D. CYP2D6 allele frequency in European Caucasians, Asians, Africans and their descendants. Pharmacogenomics 3, 229-243 (2002).

17. Gaedigk, A., Sangkuhl, K., Whirl-Carrillo, M., Klein, T. \& Leeder, J. S. Prediction of CYP2D6 phenotype from genotype across world populations. Genet. Med. 19, 69-76 (2017)

18. Aklillu, E. et al. Frequent distribution of ultrarapid metabolizers of debrisoquine in an Ethiopian population carnying duplicated and multiduplicated functional CYP2D6 alleles. J. Pharmacol. Exp. Ther. 278, 441-446 (1996).

19. Fricke-Galindo, I. et al. Interethnic variation of CYP2C19 alleles, 'predicted' phenotypes and 'measured' metabolic phenotypes across world populations. Pharmacogenomics J. 16, 113-123 (2016).

20. Cai, W.-M. et al. CYP2D6 genetic variation in healthy adults and psychiatric African-American subjects: implications for clinical practice and genetic testing. Pharmacogenomics J. 6, 343-350 (2006).

21. Gaedigk, A. et al. Identification and characterization of novel sequence variations in the cytochrome P4502D6 (CYP2D6) gene in African Americans. Pharmacogenomics J. 5, 173-182 (2005).

22. Hicks, J. K. et al. Clinical pharmacogenetics implementation consortium guideline for CYP2D6 and CYP2C19 genotypes and dosing of tricyclic antidepressants. Clin. Pharmacol. Ther. 93, 402-408 (2013).

23. Hicks, J. K. et al. Clinical pharmacogenetics implementation consortium (CPIC) guideline for CYP2D6 and CYP2C19 genotypes and dosing of selective serotonin reuptake inhibitors. Clin. Pharmacol. Ther. 98, 127-134 (2015).

24. Swen, J. J. et al. Pharmacogenetics: from bench to byte. Clin. Pharmacol. Ther. 83, 781-787 (2008)

25. Swen, J. J. et al. Pharmacogenetics: from bench to byte-an update of guidelines. Clin. Pharmacol. Ther. 89, 662-673 (2011).

26. LLerena, A. et al. Interethnic variability of CYP2D6 alleles and of predicted and measured metabolic phenotypes across world populations. Expert Opin. Drug Metab. Toxicol. 10, 1569-1583 (2014).

27. Rosenblat, J. D., Lee, Y. \& Mclntyre, R. S. Does pharmacogenomic testing improve clinical outcomes for major depressive disorder? J. Clin. Psychiatry 78, 720-729 (2017).

28. Koopmans A. B. et al. No effect of dose adjustment to the CYP2D6 genotype in patients with severe mental illness. Front. Psychiatry 9, 349 (2018),

29. Herbild, L., Andersen, S. E., Werge, T., Rasmussen, H. B. \& Jürgens, G. Does pharmacogenetic testing for CYP450 2D6 and 2C19 among patients with diagnoses within the schizophrenic spectrum reduce treatment costs? Basic Clin. Pharmacol. Toxicol. 113, 266-272 (2013).

30. Borden, B. A. et al. Assessment of provider-perceived barriers to clinical use of pharmacogenomics during participation in an institutional implementation study. Pharmacogenet. Genomics 29, 31-38 (2019).

31. Nurnberger, J. I. et al. What should a psychiatrist know about genetics? J. Clin. Psychiatry 80, e144-e151 (2018).

32. Salm, M. et al. Use of genetic tests among neurologists and psychiatrists: knowledge, attitudes, behaviors, and needs for training. J. Genet. Couns. 23, 156-163 (2014).

33. Simoons, M., Mulder, H., Schoevers, R. A., Ruhé, H. G. \& van Roon, E. N. Availability of CYP2D6 genotyping results in general practitioner and community pharmacy medical records. Pharmacogenomics 18, 843-851 (2017).

34. Hamilton, S. P. The promise of psychiatric pharmacogenomics. Biol. Psychiatry 77, 29-35 (2015).

35. Chou, W. H. et al. Extension of a pilot study: impact from the cytochrome P450 2D6 polymorphism on outcome and costs associated with severe mental illness. J. Clin. Psychopharmacol. 20, 246-251 (2000).

36. Ruaño, G. et al. Length of psychiatric hospitalization is correlated with CYP2D6 functional status in inpatients with major depressive disorder. Biomark. Med. 7, 429-439 (2013).

37. Spina, E. \& de Leon, J. Clinical applications of CYP genotyping in psychiatry. J. Neural Transm. 122, 5-28 (2015).

38. Almoguera, B. et al. CYP2D6 poor metabolizer status might be associated with better response to risperidone treatment. Pharmacogenet. Genomics $\mathbf{2 3}$, 627-630 (2013).

39. Kaur, G. et al. Identification of genetic correlates of response to risperidone: findings of a multicentric schizophrenia study from India. Asian J. Psychiatr. 29, 174-182 (2017). 
40. Moher, D., Liberati, A., Tetzlaff, J. \& Altman, D. G. Preferred reporting items for systematic reviews and meta-analyses: the PRISMA statement. PLoS Med. $\mathbf{6}$, e1000097 (2009).

41. Liberati, A. et al. The PRISMA statement for reporting systematic reviews and meta-analyses of studies that evaluate health care interventions: explanation and elaboration. PLoS Med. 6, e1000100 (2009).

42. Gaedigk, A. et al. The evolution of PharmVar. Clin. Pharmacol. Ther. 105, cpt.1275 (2019)

43. Gaedigk, A. et al. The Pharmacogene Variation (PharmVar) Consortium: incorporation of the Human Cytochrome P450 (CYP) Allele Nomenclature Database. Clin. Pharmacol. Ther. 103, 399-401 (2018).

44. Rosenberg, N. A. et al. Clines, clusters, and the effect of study design on the inference of human population structure. PLoS Genet. 1, 0660-0671 (2005).

45. Suarez-Kurtz, G. Pharmacogenomics in admixed populations. Trends Pharmacol. Sci. 26, 196-201 (2005)

46. Parra, F. C. et al. Color and genomic ancestry in Brazilians. Proc. Natl Acad. Sci. USA 100, 177-182 (2003).

47. Caudle, K. E. et al. Standardizing terms for clinical pharmacogenetic test results: consensus terms from the Clinical Pharmacogenetics Implementation Consortium (CPIC). Genet. Med. 19, 215-223 (2017).

48. Sistonen, J. et al. CYP2D6 worldwide genetic variation shows high frequency of altered activity variants and no continental structure. Pharmacogenet. Genomics 17, 93-101 (2007).

49. Wendt, F. R., Pathak, G., Sajantila, A., Chakraborty, R. \& Budowle, B. Global genetic variation of select opiate metabolism genes in self-reported healthy individuals. Pharmacogenomics J. 18, 281-294 (2018).

50. Rasmussen, J. O. et al. CYP2D6 gene test in psychiatric patients and healthy volunteers. Scand. J. Clin. Lab. Invest. 66, 129-136 (2006).

51. Naranjo, M.E. G. et al. Interethnic variability in CYP2D6, CYP2C9, and CYP2C19 genes and predicted drug metabolism phenotypes among 6060 Ibero- and Native Americans: RIBEF-CEIBA Consortium Report on Population Pharmacogenomics. OMICS 22, 575-588 (2018).

52. Vicente, J. et al. Genetic polymorphisms of CYP2C8, CYP2C9 and CYP2C19 in Ecuadorian Mestizo and Spaniard populations: a comparative study. Mol. Biol. Rep. 41, 1267-1272 (2014).

53. Mirzaev, K. B. et al. Genetic polymorphisms of cytochrome P450 enzymes and transport proteins in a Russian population and three ethnic groups of Dagestan. Genet. Test. Mol. Biomark. 21, 747-753 (2017).

54. Payan, M., Tajik, N., Rouini, M. R. \& Ghahremani, M. H. Genotype and allele frequency of CYP2C19*17 in a healthy Iranian population. Med. J. Islam Repub. Iran 29, 269 (2015).

55. Riaz, S. et al. Genetic polymorphism of CYP2C19 in Pakistani population. Iran. J. Pharm. Res. 18, 1097-1102 (2019).

56. Deshpande, N. et al. Rapid and ultra-rapid metabolizers with CYP2C19*17 polymorphism do not respond to standard therapy with proton pump inhibitors. Meta Gene 9, 159-164 (2016).

57. Ohkubo, T., Suno, M., Sugawara, K. \& Motomuro, S. Graphic roots of CYP2C19 genetic polymorphism in Japanese population (preliminary report). Int Congr. Ser. 1244, 63-67 (2002).

58. Zuo, J., Xia, D., Jia, L. \& Guo, T. Genetic polymorphisms of drugmetabolizing phase I enzymes CYP3A4, CYP2C9, CYP2C19 and CYP2D6 in Han, Uighur, Hui and Mongolian Chinese populations. Pharmazie 67 639-644 (2011).

59. Yin, S.-J. et al. Differences in genotype and allele frequency distributions of polymorphic drug metabolizing enzymes CYP2C19 and CYP2D6 in mainland Chinese Mongolian, Hui and Han populations. J. Clin. Pharmacol. Ther. 37, 364-369 (2012).

60. Shalia, K. K., Shah, V. K., Pawar, P., Divekar, S. S. \& Payannavar, S. Polymorphisms of MDR1, CYP2C19 and P2Y12 genes in Indian population: effects on clopidogrel response. Indian Heart J. 65, 158-167 (2013).

61. Anichavezhi, D., Chakradhara Rao, U. S., Shewade, D. G., Krishnamoorthy, R. \& Adithan, C. Distribution of CYP2C19*17 allele and genotypes in an Indian population. J. Clin. Pharmacol. Ther. 37, 313-318 (2012).

62. Afsar, N. A. et al. Implications of genetic variation of common drug metabolizing enzymes and $\mathrm{ABC}$ transporters among the Pakistani population. Sci. Rep. 9, 7323 (2019)

63. Riaz, S. et al. Association of CYP2C19* 2 and * 17 genetic variants with hypertension in Pakistani population. Trop. J. Pharmacol. Res. 18, 851-855 (2019).

64. Hashemizadeh, Z., Malek-Hosseini, S. A. \& Badiee, P. Prevalence of CYP2C19 genetic polymorphism among normal people and patients with hepatic diseases. Int. J. Organ Transpl. Med. 9, 27-33 (2018).
65. Dehbozorgi, M. et al. Prevalence of the CYP2C19*2 (681 G>A), *3 (636 G>A) and *17 (-806>T) alleles among an Iranian population of different ethnicities. Mol. Med. Rep. 17, 4195-4202 (2018).

66. Ortega-Vázquez, A. et al. CYP2C9, CYP2C19, ABCB1 genetic polymorphisms and phenytoin plasma concentrations in Mexican-Mestizo patients with epilepsy. Pharmacogenomics J. 16, 286-292 (2016).

67. de Andrés, F., Sosa-Macías, M., Ramos, B. P. L., Naranjo, M.E. G. \& LLerena, A. CYP450 genotype/phenotype concordance in Mexican Amerindian indigenous populations-where to from here for global precision medicine? OMiCS 21, 509-519 (2017).

68. Favela-Mendoza, A. F. et al. Correspondence between the CYP2C19 and CYP3A4 genotypes with the inferred metabolizer phenotype by omeprazole administration in Mexican healthy children. J. Clin. Pharmacol. Ther. 43, 656-663 (2018).

69. Céspedes-Garro C. et al. Relevance of the ancestry for the variability of the drug-metabolizing enzymes CYP2C9, CYP2C19 and CYP2D6 polymorphisms in a multiethnic Costa Rican population. Rev. Biol. Trop. 64, 1067-1076 (2016).

70. Ingelman-Sundberg, M., Sim, S. C., Gomez, A. \& Rodriguez-Antona, C. Influence of cytochrome P450 polymorphisms on drug therapies: pharmacogenetic, pharmacoepigenetic and clinical aspects. Pharmacol. Ther. 116 496-526 (2007).

71. Huddart, R. et al. Standardized biogeographic grouping system for annotating populations in pharmacogenetic research. Clin. Pharmacol. Ther. 105 1256-1262 (2019).

72. Zhang, F. \& Finkelstein, J. Inconsistency in race and ethnic classification in pharmacogenetics studies and its potential clinical implications. Pharmgenomics Pers. Med. 12, 107-123 (2019).

73. Riccardi, L. N. et al. CYP2D6 genotyping in natives and immigrants from the Emilia-Romagna region (Italy). Genet. Test. Mol. Biomark. 15, 801-806 (2011).

74. Dodgen, T. M. et al. Introduction of the AmpliChip CYP450 Test to a South African cohort: a platform comparative prospective cohort study. BMC Med. Genet. 14, 20 (2013).

75. Shah, R. R. Pharmacogenetics and precision medicine: is inflammation a covert threat to effective genotype-based therapy? Ther. Adv. Drug Saf. 8 267-272 (2017).

76. Shah, R. R. \& Gaedigk, A. Precision medicine: does ethnicity information complement genotype-based prescribing decisions? Ther. Adv. Drug Saf. 9, 45-62 (2018).

77. Berm, E., Kok, R., Hak, E. \& Wilffert, B. Relation between CYP2D6 genotype, phenotype and therapeutic drug concentrations among nortriptyline and venlafaxine users in old age psychiatry. Pharmacopsychiatry 49, 186-190 (2016).

78. Preskorn, S. H. et al. Cytochrome P450 2D6 phenoconversion is common in patients being treated for depression. J. Clin. Psychiatry 74, 614-621 (2013).

79. de Andrés, F., Terán, S., Hernández, F., Terán, E. \& LLerena, A. To genotype or phenotype for personalized medicine? CYP450 drug metabolizing enzyme genotype-phenotype concordance and discordance in the Ecuadorian population. OMICS 20, 699-710 (2016).

80. Torres, U. S., Portela-Oliveira, E., Borgwardt, S. \& Busatto, G. F. Structural brain changes associated with antipsychotic treatment in schizophrenia as revealed by voxel-based morphometric MRI: an activation likelihood estimation meta-analysis. BMC Psychiatry 13, 342 (2013).

81. Zutshi, D., Cloud, L. J. \& Factor, S. A. Tardive syndromes are rarely reversible after discontinuing dopamine receptor blocking agents: experience from a universitybased movement disorder clinic. Tremor Other Hyperkinet. Mov, 4, 1-9 (2014).

82. Gaedigk, A. et al. The CYP2D6 activity score: translating genotype information into a qualitative measure of phenotype. Clin. Pharmacol. Ther. 83, 234-242 (2008).

83. Kirchheiner, J., Meineke, I., Müller, G., Roots, I. \& Brockmöller, J. Contributions of CYP2D6, CYP2C9 and CYP2C19 to the biotransformation of E- and Zdoxepin in healthy volunteers. Pharmacogenetics 12, 571-580 (2002).

84. Chang, M., Tybring, G., Dahl, M.-L. \& Lindh, J. D. Impact of cytochrome P450 2C19 polymorphisms on citalopram/escitalopram exposure: a systematic review and meta-analysis. Clin. Pharmacokinet. 53, 801-811 (2014).

85. Steimer, W. et al. Amitriptyline or not, that is the question: pharmacogenetic testing of CYP2D6 and CYP2C19 identifies patients with low or high risk for side effects in amitriptyline therapy. Clin. Chem. 51, 376-385 (2005).

86. Laika, B., Leucht, S., Heres, S. \& Steimer, W. Intermediate metabolizer: increased side effects in psychoactive drug therapy. The key to costeffectiveness of pretreatment CYP2D6 screening? Pharmacogenomics J. 9 395-403 (2009). 
87. Hicks, J. et al. Clinical pharmacogenetics implementation consortium guideline (CPIC) for CYP2D6 and CYP2C19 genotypes and dosing of tricyclic antidepressants: 2016 update. Clin. Pharmacol. Ther. 102, 37-44 (2017).

88. González, I. et al. Relation between CYP2D6 phenotype and genotype and personality in healthy volunteers. Pharmacogenomics 9, 833-840 (2008).

89. Babalola, C. P. et al. Cytochrome P450 CYP2C19 genotypes in Nigerian sicklecell disease patients and normal controls. Pharmacogenetics 35, 471-477 (2010).

90. Barañska, M., Dziankowska-bartkowiak, B., Waszczykowska, E., Rychlik-sych, M. \& Skrêtkowicz, J. Significance of genetic polymorphism of CYP2D6 in the pathogenesis of systemic sclerosis. Pharmacol. Rep. 64, 336-342 (2012).

91. Singh, M. et al. Polymorphism in environment responsive genes and association with Parkinson disease. Mol. Cell. Biochem. 312, 131-138 (2008).

92. Brown, M. A. et al. Polymorphisms of the CYP2D6 gene increase susceptibility to ankylosing spondylitis. Hum. Mol. Genet. 9, 1563-1566 (2000).

93. Ladona, M. et al. CYP2D6 genotypes in Spanish women with breast cancer. Cancer Lett. 99, 23-28 (1996).

94. Singh, H., Lata, S. \& Gangakhedkar, R. R. Prevalence of CYP2D6 * 4 1934G/A polymorphism in Western Indian HIV patients. J. Pathol. Microbiol. Immunol. 126, 842-851 (2018).
95. Del Tredici, A. L. et al. Frequency of CYP2D6 alleles including structural variants in the United States. Front. Pharmacol. 9, 305 (2018).

96. Griman, P. et al. CYP2D6 gene variants in urban/admixed and Amerindian populations of Venezuela: pharmacogenetics and anthropological implications. Ann. Hum. Biol. 39, 137-142 (2012).

97. Yousef, A.-M. et al. Allele and genotype frequencies of the polymorphic cytochrome P450 genes (CYP1A1, CYP3A4, CYP3A5, CYP2C9 and CYP2C19) in the Jordanian population. Mol. Biol. Rep. 39, 9423-9433 (2012).

98. Budd, W. T. et al. Next generation sequencing reveals disparate population frequencies among cytochrome P450 genes: clinical pharmacogenomics of the CYP2 family. Int. J. Comput. Biol. Drug Des. 9, 54 (2016).

99. Pratt, V. M. et al. Characterization of 107 Genomic DNA Reference Materials for CYP2D6, CYP2C19, CYP2C9, VKORC1, and UGT1A1. J. Mol. Diagn. 12 835-846 (2010).

100. Banda, Y. et al. Characterizing race/ethnicity and genetic ancestry for 100,000 subjects in the Genetic Epidemiology Research on Adult Health and Aging (GERA) cohort. Genetics 200, 1285-1295 (2015).

101. Pratt, V. M. et al. Recommendations for clinical CYP2C19 Genotyping allele selection. J. Mol. Diagn. 20, 269-276 (2018). 